ISSN 1936-5098

CAE Working Paper \#09-10

\title{
Pet Overpopulation: An Economic Analysis
}

by

Stephen Coate

and

Brian Knight

August 2009 
August 2009

\title{
Pet Overpopulation: An Economic Analysis*
}

\begin{abstract}
This paper considers the problem of pet overpopulation. It develops a tractable dynamic model whose positive predictions square well with key features of the current U.S. market for pets. The model is used to understand, from a welfare economic perspective, the sense in which there is "overpopulation" of pets and the underlying causes of the problem. The paper also employs the model to consider what policies might be implemented to deal with the problem. A calibrated example is developed to illustrate these corrective policies and quantify the welfare gains they produce.
\end{abstract}

Stephen Coate

Department of Economics

Cornell University

Ithaca NY 14853

sc163@cornell.edu

Brian Knight

Department of Economics

Brown University

Providence RI 02912

brian_knight@brown.edu

*We thank Jon Conrad, David Harris, Jan Scarlett, and Sharon Tennyson for useful discussions. 


\section{Introduction}

The market for companion animals in the U.S. is important economically and socially. About 63 percent of households own pets, and they spend substantial amounts feeding and taking care of them. ${ }^{1}$ Pets generate significant psychic benefits for their owners. Indeed, according to a recent survey, a majority of owners view their pets as members of their families (APPMA, 2005). However, there are reasons to believe that the market for pets produces less than socially optimal outcomes. The key problem is that of pet overpopulation. There is little demand for older pets relinquished by their owners due to changes in personal circumstances, such as job loss, foreclosure, divorce, or health problems. Even owners with mixed breed puppies or kittens often have trouble finding homes for them. As a consequence, an estimated 11 million dogs and 9 million cats enter animal shelters annually, and 5 million dogs and 6 million cats end up being euthanized. At the same time, new owners are buying pure breed puppies and kittens for significant sums of money.

The problem of pet overpopulation has generated much public interest and discussion about appropriate policy responses. The most common approach is to work on the supply side of the market by encouraging, or even requiring, the neutering and spaying of pets. The California legislature, for example, recently considered a mandatory neutering policy, which would have represented the first such state-level policy. ${ }^{2} \quad$ An alternative, but largely untested, strategy is to work on the demand side, by, for example, taxing the sale of pure breed puppies or kittens, or otherwise encouraging citizens to adopt shelter dogs or cats.

The economics literature has been surprisingly silent on the problem of pet overpopulation and, indeed, on the economics of pets more generally. ${ }^{3}$ In particular, the literature has not provided an understanding of the performance of the market for companion animals and the appropriate role for government intervention. Given their long-lived nature, it is natural to think of pets as

\footnotetext{
1 According to AVMA (2002), dog owners report spending $\$ 261$ on average during 2001 on veterinary services alone, and cat owners reported spending $\$ 157$. In terms of food, dog owners spent $\$ 241$ on average during 2004, and cat owners spent $\$ 185$ (APPMA, 2005).

2 This proposal was eventually shelved due to opposition from breeders and other constituencies (Associated Press, July 11, 2007).

3 A few papers have explored the demand for pets using survey data (see, for example, Endenburg, 't Hart, and Bouw (1994) and Hirschman (1994)). These papers seek to understand the reasons why consumers do or do not keep pets. Schwarz, Troyer, and Walker (2007) explore the timing of household spending on pets over the life cycle and its correlation with the number and age of children. Their theoretical framework is a life cycle model in which households simultaneously choose children and pets. They show empirically that pets are a substitute for very young children and a complement with older children. In all phases of the life cycle, a larger number of children is associated with a lower number of pets.
} 
durable goods. However, there are important differences between companion animals and other durable goods. Perhaps the key one is that, due to bonding, consumers care about the pets they have owned. This has two consequences. First, consumers obtain a higher payoff from an older animal if they owned it when young. Second, owners who have to relinquish their pets suffer psychic costs if they do not find homes and are euthanized. A further distinctive feature is that the future population of pets is "produced" by the current population. Moreover, the production costs of mixed breed pets may actually be negative since production can only be prevented by costly spaying and neutering.

This paper presents a model of the market that captures some of its complexities and uses it to analyze market performance and the scope for beneficial government intervention. The equilibrium of the model qualitatively matches key features of the markets for dogs and cats in the U.S.. Old pets relinquished by their owners are euthanized, while pure breed puppies and kittens are sold for a positive price. Mixed breed puppies and kittens are available for free; some are adopted and some euthanized. The euthanization of pets makes the equilibrium inefficient and it is in this sense that the market creates pet overpopulation. The inefficiency stems from the assumption that consumers care about the animals they have owned. A common pool externality arises from the fixed number of "good homes" to which young or relinquished older pets can be allocated. When making their breeding or spaying decisions, owners do not take into account that their puppies or kittens crowd out homes for other needy pets. Restoring efficiency requires a combination of taxes on young pets and subsidies for spaying.

A calibrated example is developed to illustrate the nature of the optimal corrective policies and to quantify the magnitude of potential welfare gains. The parameters of the model are chosen so the equilibrium quantitatively matches the market for dogs in the U.S.. The optimal taxes on young dogs are large, exceeding the prices currently paid for pure breed puppies. The aggregate welfare gains are also sizable, in the $\$ 16$ to $\$ 21$ billion range. These estimates suggest that the problem of pet overpopulation deserves serious policy consideration.

The paper contributes to an extensive literature on the pet overpopulation problem, mostly written by veterinarians and psychologists. This literature seeks to understand how many pets are taken to shelters, why they are taken, and their fate after arrival. It is also interested in the determinants of spaying and neutering decisions, and the dynamics of pet populations more generally. While this literature provides vital information about the problem, it does not offer an analytical 
framework in which to consider optimal policy responses. The one exception is Frank (2004) who develops and calibrates a mathematical population flow model of the dog population. He then uses this model to assess the cost effectiveness of different strategies for reducing euthanization rates, including spaying subsidies and programs designed to encourage adoption, such as taxes on non-shelter dogs. ${ }^{4}$ Viewing these programs as substitutes, he finds that spaying subsidies are the most cost effective. Allowing for synergies, he concludes that spaying subsidies and programs designed to encourage adoption work well in tandem. Frank's work differs from ours in that his model is ecological rather than economic. Thus, there are no prices, and demand and supply behavior is not derived from the decisions of optimizing agents. ${ }^{5}$ In addition, his model does not distinguish between young and old, or pure and mixed breed dogs. On the other hand, his model better handles some of the complexities of the market, including the role of pet stores and feral populations. Given all this, we see our analysis as complementary to Frank's work.

The paper also contributes to the literature on the economics of animals, particularly that strand developing dynamic models of animal populations. One branch of this work focuses on wild animals that are hunted by humans for meat, fur, or other by-products. Examples are beavers (Carlos and Lewis (1993)), buffalo (Taylor (2007)), elephants (Kremer and Morcom (2000)), fish (Gordon (1954)), and whales (Allen and Keay (2004)). In sharp contrast to pets, the key concern for these animals is overexploitation and the resulting problems of underpopulation and potential extinction. A second branch of this literature focuses on farm animal populations (see, for example, Rosen (1987) and Rosen, Murphy and Scheinkman (1994)). The main interest lies in understanding the dynamics of stocks of farm animals. Given this research, it seems natural to investigate the dynamics of companion animal populations and our paper does exactly this.

The organization of the remainder of the paper is as follows. Section 2 provides background on the market for pets and Section 3 describes the model. Section 4 characterizes market equilibrium and Section 5 explores efficiency. Section 6 identifies policy interventions that can improve welfare and Section 7 develops a calibrated example to illustrate these policies. Section 8 concludes.

\footnotetext{
${ }^{4}$ In addition to reducing euthanasia rates, Frank (2001) also considers other objectives depending upon animal welfare. For example, he investigates which programs would minimize animal suffering, which, according to his definition, includes not only the suffering for those animals euthanized but also the suffering associated with living in overcrowded shelters or the difficulties of surving in the wild.

5 The effectiveness of different strategies is assessed by tracing out the implications of changing certain key behavioral parameters in the model (for example, the fraction of owners who spay or neuter their dogs, or the fraction of owners who adopt pets from shelters) and using survey data to speculate on how costly it would be to generate the behavioral change in question.
} 


\section{Background}

Dogs and cats are the most popular type of pets in the U.S. with 39 percent of households owning dogs and 34 percent owning cats (APPMA, 2005). Among dog-owning households, the most common number is one (60 percent) followed by two (25 percent), with an average of 1.7. Catowning households, by contrast, are more likely to own multiple cats, with about half owning more than one (AVMA, 2002). A key distinction in the market for pets is pure versus mixed breed, with about half of dog-owning households in possession of a pure breed (New et. al., 2000). Pure breed cats, by contrast, are relatively rare, with over 90 percent of cat-owning households in possession of a mixed breed.

Owners typically acquire their pets as puppies or kittens. ${ }^{6}$ Common suppliers include breeders, owners who advertise in the classifieds, animal shelters, friends and relatives, and pet stores. In terms of the market for puppies, prices paid by owners vary significantly by breed (APPMA, 2005). On average, owners spent almost $\$ 500$ for pure breeds. The market conditions for mixed breed dogs are less clear. In some regions, there is an excess supply of mixed breed puppies, and households can adopt them for free from animal shelters. In others, shelters report having very few puppies, suggesting that mixed breeds are being sold at a positive price (DiGiacomo, Arluke, and Patronek, 1998). However, even in this case, the prices paid are well below those for pure breeds. Pure breed kittens can also be expensive, with prices of $\$ 300$ and up for popular breeds. Mixed breed kittens, however, are typically available for free adoption at animal shelters.

Pet ownership is regulated at the local level, with considerable variation both across and within states. Local ordinances limit the number and type of animals that can be owned, and restrict the way in which households may keep their pets. ${ }^{7}$ In most communities, dog owners are required to purchase licenses for their animals. Licenses are typically in the $\$ 10-\$ 50$ range for an intact dog, with a 50 percent discount for spaying or neutering, and must be renewed annually. ${ }^{8}$ In certain communities, cat licenses are also required. Revenue from licenses is used to finance animal control

\footnotetext{
${ }^{6}$ According to a survey in an Indiana community, 84 percent of dogs and cats were obtained when they were less than one year old (Patronek, Beck, and Glickman, 1997).

7 For example, some communities limit the number of dogs that can be owned by a household (e.g., less than four), others ban ownership of certain breeds (e.g., pit bulls), and almost all prohibit the roaming of dogs.

8 There is little information on the fraction of households who actually obtain licenses for their pets. In Frank's (2001) survey of a region of upstate New York, 72 percent of dog owners claimed to have obtained a license. Enforcement is usually done by a public official who visits homes to check if pets have tags.
} 
operations and to provide (or subsidize) animal shelters.

Animal shelters, which are typically either local governmental agencies or non-profit organizations, handle a huge number of unwanted pets. According to the Shelter Statistics Survey, 1994-1997, which surveyed roughly 1000 shelters, over 2.3 million dogs and 1.8 million cats entered these shelters on an annual basis. ${ }^{9}$ Assuming that the respondents represent a random sample of the roughly 5000 national shelters, this implies that over 11 million dogs and around 9 million cats enter shelters annually. In terms of their sources, puppies and kittens, typically mixed-breed, are brought in by owners who cannot find them homes, older pets are brought in by owners who can no longer take care of them, and strays, which include those dumped by their owners, are brought in by animal control. ${ }^{10}$

Pets entering a shelter are eventually either adopted, returned to their owners, or euthanized. For dogs, the most likely outcome is euthanasia (56 percent) with 25 percent adopted and 16 percent being returned to their owners. Cats are also adopted at a rate of about 25 percent. Very few cats are returned to their owners, however, and over 70 percent of cats entering shelters are euthanized. Again, assuming that the survey represents a random sample of shelters, this implies that over 5 million dogs and 6 million cats are euthanized annually.

While there is no national information on which types of dogs and cats tend to be euthanized versus adopted in shelters, some anecdotal evidence is available. Using data from a shelter in Sacremento County, California, Lepper, Kass, and Hart (2002) find that age is a key determinant of euthanasia. Puppies, defined as less than 1 year old, were more than two times as likely to be adopted as dogs 1 to 2 years old and about three times as likely as dogs 3 to 5 years old. Dogs over 5 years of age were very unlikely to be adopted, with adoption rates just 2 percent of those of puppies. For cats, kittens were about 20 times more likely to be adopted than older cats, defined as over 5 years old.

An important issue is why owners relinquish their older pets to shelters. Diacomo, Arluke and Patronek's (1998) case study suggests that, for most relinquishing owners, giving up a pet is

\footnotetext{
9 These data were obtained from the website http://www.petpopulation.org/statsurvey.html (accessed June 2009).

10 According to the Shelter Statistics Survey, 38 percent of pets entering shelters were submitted by animal control, 30 percent were relinquished by owners, and 32 percent were classified as either other or unknown sources. In terms of the types of pets relinquished by owners, data from another survey suggests that pets in shelters, relative to pets in households, tend to be younger, including a large number of puppies and kitttens, and of mixed breed status (New et al, 2000).
} 
very traumatic. Survey data suggest that both unforeseen changes in the owner's situation and behavioral issues with the pet are key causes of relinquishment. The two most common reasons offered for giving up dogs were moving and issues with landlords. Behavioral problems, such as illness and biting, were also important, but lower on the list of reasons. For cats, allergies and moving are common reasons for relinquishment. ${ }^{11}$

In response to the significant euthanasia of both cats and dogs, several policies have been proposed and implemented. Pets adopted from shelters, for example, must typically be spayed or neutered. As noted above, licenses are usually set at lower prices for altered pets. The discount can be substantial as in Chicago, Illinois, where a license costs $\$ 5$ for an altered dog versus $\$ 50$ for an intact dog. A few cities, such as Santa Cruz, California, have established mandatory neutering policies. Despite these efforts, a large number of pets remain intact. According to a recent survey of pet-owning households conducted by New et. al. (2000), only 60 percent of dogs and 78 percent of cats have been neutered or spayed.

In conclusion, we want to highlight four key features of the market for pets that are crucial for the development of the model. First, there is an important distinction between younger and older pets, with strong demand for younger pets and much weaker demand for relinquished older pets. Second, among younger pets, there is strong demand for pure breeds, as evidenced by the high prices for pure breed puppies and kittens. Third, for young mixed breed pets, excess supply, zero price, and euthanasia are very common. Finally, changes in personal circumstances beyond the control of the owner are often key factors in the relinquishment of pets.

\section{The model}

We develop a dynamic partial equilibrium model of the market for a single species of pet, such as dogs or cats.

\subsection{Pets and owners}

Time is discrete and indexed by $t=1, \ldots, \infty$. There are a continuum of infinitely-lived potential pet owners of size $1 / \alpha$ where $\alpha \in(0,1)$. Each owner can own at most one pet in any period. Moreover, each owner is able to own a pet in any period with probability $\alpha$, implying that the size of the population able to own a pet in any period is 1 . This uncertainty is designed to capture

\footnotetext{
11 This information is taken from the website http://www.petpopulation.org/exploring.pdf (accessed June 2009).
} 
random events (divorce, job loss, health problems, etc) which impact the ability to own a pet. All owners discount future payoffs at rate $\delta$.

Pets come in two varieties, pure and mixed breed, indexed by $i \in\{p, m\}$, and live for at most two periods. In their first period of life, pets are young and, in the second, they are old. A pet's age is indexed by $a \in\{y, o\}$. Young pets can die of natural causes before they get old. The probability that a young pet reaches old age is $\eta<1$.

Potential pet owners are divided into two groups, low and high types, indexed by $K \in\{L, H\}$. The fraction of $K$ types in the population is $\gamma_{K}$. High types have a preference for pure breeds and low types do not. Specifically, high types enjoy a period benefit $\beta$ from owning a young mixed breed and $\beta+\theta$ from a young pure breed, while low types obtain $\beta$ from either. The benefits potential owners get from owning an old pet are the same as those from owning a young pet if they owned the pet when young. Otherwise, benefits are deflated by the parameter $\lambda \in(0,1)$. Thus, high types get a benefit $\lambda(\beta+\theta)$ from an old pure breed they did not own when young and $\lambda \beta$ from an old mixed breed, while low types get $\lambda \beta$ from either. This deflation captures the idea that owning an old pet is not as much fun as a young one, although a pet owned when young creates a bond which compensates for this.

When young, pets can have offspring. There are $x$ offspring in a litter. Young pets can be spayed at cost $c_{s}$. A young mixed breed that is not spayed will produce a litter of mixed breed offspring with probability $\varphi$. Pure breeds can be bred at cost $r$ to produce a litter of pure breed offspring. However, a young pure breed that is neither spayed nor bred will also produce a litter of mixed breed offspring with probability $\varphi$. This captures the idea that, in the real world, accidental mating is unlikely to be with another pure breed of the exact same type and hence the result will be mixed breed offspring. Any owner whose pet has a litter incurs a cost $c_{l}$ as a result of the event. This captures the costs of providing care to puppies or kittens and the disruption they cause (whining, chewing, scratching, accidents, etc).

If the pet population exceeds the number of owners, there will be surplus pets and such pets must be euthanized. Euthanization entails a resource cost $\rho$ and a psychic cost bourne by the individual who previously owned the pet. This psychic cost is $\xi_{y}$ for a young pet and $\xi_{o}$ for an old pet where $\xi_{o} \geq \xi_{y}$. The inequality is motivated by the idea that the owner of an old pet will have a stronger bond to it. 


\subsection{Allocation and production plans}

The economic problem is to determine the intertemporal allocation and production of pets. In each period, there will be a population of young pets that need to be allocated among new owners, defined as those who can own a pet but do not currently own an old one. ${ }^{12}$ There will also be a population of old pets whose owners are no longer able to keep them and these relinquished old pets must also be allocated among new owners. ${ }^{13}$ If there are more pets than available homes, the surplus pets will be euthanized. In addition to all this, next period's population of young pets must be determined by today's breeding and spaying decisions.

Exploring this problem formally requires a notation that accounts for the populations of pets in each period and their allocation across owner types. The population of young pure and mixed breeds at the beginning of period $t$ will be denoted by $\mathbf{y}_{t}=\left(y_{p t}, y_{m t}\right)$. This population is determined by the breeding and spaying decisions in period $t-1$. The population of old pets at the beginning of period $t$ can be described by the characteristics of their owners. To understand this, recall that young pets without owners in period $t-1$ are euthanized and hence all old pets at the beginning of period $t$ will be owned by the owners who owned them when young. Let $K_{i t}^{o}$ denote the number of $K$ type owners who own old pets of breed $i$ at the beginning of period $t$. Let $K_{t}^{o}=K_{p t}^{o}+K_{m t}^{o}$ denote the total number of $K$ type owners of old pets and let $\mathbf{K}_{t}^{o}$ denote the vector $\left(K_{p t}^{o}, K_{m t}^{o}\right)$. The population of old pets at the beginning of period $t$ can then be described by the vector $\left(\mathbf{H}_{t}^{o}, \mathbf{L}_{t}^{o}\right)$. In particular, the population of old pets of breed $i$ is $H_{i t}^{o}+L_{i t}^{o}$.

In any period, a fraction $1-\alpha$ of old pet owners will be unable to keep them. Thus, in period $t$, old owners will relinquish $(1-\alpha)\left(H_{p t}^{o}+L_{p t}^{o}\right)$ pure breeds and $(1-\alpha)\left(H_{m t}^{o}+L_{m t}^{o}\right)$ mixed breeds. The young and relinquished old pets must be allocated among the new owners. The number of $K$ type new owners in period $t$ will be $\gamma_{K}-\alpha K_{t}^{o}$. This reflects the fact that the total number of $K$ types who can own pets in each period is $\gamma_{K}$, and the number who will end up being able to keep their old pets is $\alpha K_{t}^{o}$. Let $K_{a i t}^{n}$ denote the number of $K$ type new owners who are allocated a pet of age $a$ and breed $i$ in period $t$. In addition, let $\mathbf{K}_{y t}^{n}$ denote the vector $\left(K_{y p t}^{n}, K_{y m t}^{n}\right), \mathbf{K}_{o t}^{n}$ the vector $\left(K_{o p t}^{n}, K_{o m t}^{n}\right)$, and $\mathbf{K}_{t}^{n}$ the composite vector $\left(\mathbf{K}_{y t}^{n}, \mathbf{K}_{o t}^{n}\right)$.

\footnotetext{
12 A new owner can own puppies or kittens if he owned a young pet in the previous period which died after producing a litter.

13 We ignore the possibility of reallocating old pets whose owners can keep them since this will neither be socially optimal nor part of an equilibrium.
} 
In each period $t$, given the population of old pets as summarized by $\left(\mathbf{H}_{t}^{o}, \mathbf{L}_{t}^{o}\right)$ and the population of young pets $\mathbf{y}_{t}$, the economic problem is to allocate the young and relinquished old pets among the new owners by choosing $\mathbf{H}_{t}^{n}$ and $\mathbf{L}_{t}^{n}$, and to determine the population of young pets for the next period by choosing $\mathbf{y}_{t+1}$. The allocation decisions made in period $t$ will determine next period's population of old pets via the transition equation $\left(\mathbf{H}_{t+1}^{o}, \mathbf{L}_{t+1}^{o}\right)=\eta\left(\mathbf{H}_{y t}^{n}, \mathbf{L}_{y t}^{n}\right)$. This equation reflects the assumptions that young pets reach old age with probability $\eta$ and that all young pets not owned in period $t$ are euthanized. The transition equation creates an intertemporal linkage between today's allocation decisions and tomorrow's allocation problem.

The choice of $\mathbf{H}_{t}^{n}$ and $\mathbf{L}_{t}^{n}$ must respect certain feasibility constraints. ${ }^{14}$ The first is that the number of new owners allocated pets is no greater than the population of such owners; that is,

$$
K_{y p t}^{n}+K_{y m t}^{n}+K_{o p t}^{n}+K_{o m t}^{n} \leq \gamma_{K}-\alpha K_{t}^{o} \quad K \in\{L, H\} .
$$

The second class of constraints says that the number of new owners allocated each type of pet cannot exceed the total numbers available. Thus, for the young pets,

$$
L_{y i t}^{n}+H_{y i t}^{n} \leq y_{i t} \quad i \in\{p, m\}
$$

and, for the relinquished old pets,

$$
L_{\text {oit }}^{n}+H_{\text {oit }}^{n} \leq(1-\alpha)\left(L_{i t}^{o}+H_{i t}^{o}\right) \quad i \in\{p, m\} .
$$

Subtracting the left from the right hand side of these equations, gives the number of pets of each type that will be without homes and must therefore be euthanized.

An allocation and production plan $\left(\mathbf{H}_{t}^{n}, \mathbf{L}_{t}^{n}, \mathbf{y}_{t+1}\right)_{t=1}^{\infty}$ is a description of the allocation and production decisions in each period. A plan $\left(\mathbf{H}_{t}^{n}, \mathbf{L}_{t}^{n}, \mathbf{y}_{t+1}\right)_{t=1}^{\infty}$ is feasible if, given the populations of old and young pets the community begins with in period 1 (i.e., $\left(\mathbf{H}_{1}^{o}, \mathbf{L}_{1}^{o}\right)$ and $\left.\mathbf{y}_{1}\right)$ and the transition equation $\left(\mathbf{H}_{t+1}^{o}, \mathbf{L}_{t+1}^{o}\right)=\eta\left(\mathbf{H}_{y t}^{n}, \mathbf{L}_{y t}^{n}\right)$, the period $t$ choice $\left(\mathbf{H}_{t}^{n}, \mathbf{L}_{t}^{n}, \mathbf{y}_{t+1}\right)$ satisfies the feasibility constraints (1), (2), and (3) for all $t$.

\subsection{Welfare}

The particular allocation and production plan chosen will determine the (pet-related) surplus generated in the community. Let $S\left(\mathbf{H}_{t}^{o}, \mathbf{L}_{t}^{o}, \mathbf{y}_{t}, \mathbf{H}_{t}^{n}, \mathbf{L}_{t}^{n}, \mathbf{y}_{t+1}\right)$ denote the surplus accruing in period

\footnotetext{
14 The choice of $\mathbf{y}_{t+1}$ must also respect the feasibility constraints that there be sufficient young pets in period $t$ to generate the period $t+1$ populations $\mathbf{y}_{t+1}$. Since the focus of our analysis is on problems of overpopulation, we avoid the notational burden of explicitly introducing these constraints. We will also not be explicit that all variables must be non-negative, although the analysis will respect these constraints.
} 
$t$ given the initial old and young pet populations $\left(\mathbf{H}_{t}^{o}, \mathbf{L}_{t}^{o}\right)$ and $\mathbf{y}_{t}$, and the period $t$ choices $\left(\mathbf{H}_{t}^{n}, \mathbf{L}_{t}^{n}\right)$ and $\mathbf{y}_{t+1}$. This surplus comes from four different sources which we now describe.

The first source is the benefit enjoyed by owners of old pets who are able to keep them in period $t$. This is given by

$$
B_{t}^{o}=(\beta+\theta) \alpha H_{p t}^{o}+\beta \alpha\left(H_{m t}^{o}+L_{p t}^{o}+L_{m t}^{o}\right) .
$$

The first term is the benefit obtained by high types who own pure breeds and the second is the benefit obtained by mixed breed owners and low types who own pure breeds. The formula reflects the assumptions that old owners are able to keep their pets with probability $\alpha$ and that low types are indifferent between pure and mixed breeds.

The second source of surplus is the benefit experienced by new owners in period $t$. This is given by

$$
B_{t}^{n}=(\beta+\theta) H_{y p t}^{n}+\beta\left(H_{y m t}^{n}+L_{y p t}^{n}+L_{y m t}^{n}\right)+\lambda(\beta+\theta) H_{o p t}^{n}+\lambda \beta\left(H_{o m t}^{n}+L_{o p t}^{n}+L_{o m t}^{n}\right) .
$$

The first term is the benefit enjoyed by high types owning young pure breeds and the second the benefit from high types owning young mixed breeds and low types owning young pets. The final two terms are the benefits of new owners owning old relinquished pets. They reflect the assumption that owning an old pet generates less surplus for a new owner than a young pet.

The third component of surplus is the cost of euthanizing pets. As noted earlier, the number of pets of each type that will need to be euthanized, is obtained by differencing the right and left hand sides of equations (2) and (3). Thus, total euthanizing costs are

$$
E_{t}=\left(\xi_{y}+\rho\right) \sum_{i=m}^{p}\left(y_{i t}-L_{y i t}^{n}-H_{y i t}^{n}\right)+\left(\xi_{o}+\rho\right) \sum_{i=m}^{p}\left((1-\alpha)\left(L_{i t}^{o}+H_{i t}^{o}\right)-L_{o i t}^{n}-H_{o i t}^{n}\right) .
$$

The fourth and final component of surplus is the cost of breeding and spaying pets. This is given by

$$
Z_{t}=\left(\frac{y_{p t+1}}{x}\right)\left(r+c_{l}\right)+\left(\frac{y_{m t+1}}{x}\right) c_{l}+\left(H_{y p t}^{n}+L_{y p t}^{n}+H_{y m t}^{n}+L_{y m t}^{n}-\frac{y_{m t+1}}{\varphi x}-\frac{y_{p t+1}}{x}\right) c_{s} .
$$

The first and second terms reflect the direct costs of producing the young pure and mixed breeds. The third term reflects the costs of spaying the pets that are not used for breeding or for generating mixed breeds. 
Period $t$ surplus $S\left(\mathbf{H}_{t}^{o}, \mathbf{L}_{t}^{o}, \mathbf{y}_{t}, \mathbf{H}_{t}^{n}, \mathbf{L}_{t}^{n}, \mathbf{y}_{t+1}\right)$ is obtained by adding the new and old owners' benefits and subtracting the euthanization and breeding costs; i.e.,

$$
S\left(\mathbf{H}_{t}^{o}, \mathbf{L}_{t}^{o}, \mathbf{y}_{t}, \mathbf{H}_{t}^{n}, \mathbf{L}_{t}^{n}, \mathbf{y}_{t+1}\right)=B_{t}^{o}+B_{t}^{n}-E_{t}-Z_{t}
$$

Lifetime surplus in the community will be determined by the entire plan. Specifically, given the first period populations of old and young pets $\left(\left(\mathbf{H}_{1}^{o}, \mathbf{L}_{1}^{o}\right)\right.$ and $\left.\mathbf{y}_{1}\right)$ and the transition equation $\left(\mathbf{H}_{t+1}^{o}, \mathbf{L}_{t+1}^{o}\right)=\eta\left(\mathbf{H}_{y t}^{n}, \mathbf{L}_{y t}^{n}\right)$, lifetime surplus under the allocation and production plan $\left(\mathbf{H}_{t}^{n}, \mathbf{L}_{t}^{n}, \mathbf{y}_{t+1}\right)_{t=1}^{\infty}$ is given by

$$
\sum_{t=1}^{\infty} \delta^{t-1} S\left(\mathbf{H}_{t}^{o}, \mathbf{L}_{t}^{o}, \mathbf{y}_{t}, \mathbf{H}_{t}^{n}, \mathbf{L}_{t}^{n}, \mathbf{y}_{t+1}\right)
$$

\subsection{Remarks about the model}

The model incorporates many simplifying assumptions designed to keep it tractable and focused on the most salient considerations. Here we briefly identify and discuss some of the most important.

First, by assuming that all pets can have offspring, the model ignores issues of gender. This precludes, among other things, endogenizing the probability that an unspayed pet has offspring and considering the relative effectiveness of spaying versus neutering as population management strategies. The justification for this assumption is purely one of tractability: allowing for male and female pets would double the number of pet types to keep track of and also necessitate introducing owner preferences for gender. While this might be feasible, the extension would be sufficiently involved that it is best left for another paper.

Second, the model assumes that owners can own at most one pet and, moreover, that there is limited heterogeneity across potential owners in the benefits of pet ownership. These assumptions effectively make the total demand for pets perfectly inelastic. This permits a clean focus on consumer decisions of the type of pet to own (i.e., young pure breed, young mixed breed, etc) but rules out policy effects on the total number of pets owned. The assumption of at most one pet also rules out the possibility of commercial breeders who own a large number of pets and produce multiple litters of puppies or kittens which they then sell to pet stores. We do this for tractability but also because most owners purchase their dogs and cats from local small-scale breeders rather than through pet stores. ${ }^{15}$

\footnotetext{
${ }^{15}$ For example, Patronek, Beck and Glickman's (1997) survey of pet owners in St Joseph County, Indiana revealed that only $9 \%$ of dog owning households and $5 \%$ of cat owning households obtained their pets from pet stores.
} 
Third, the model assumes that the only reason an owner relinquishes an old pet is because he becomes unable to care for it. In reality, dogs and cats develop behavioral problems, such as aggression or soiling, which make them unsuitable as pets. Indeed, as discussed in Section 2, this is a major reason for owner relinquishment. Such problems can, in fact, be incorporated into the model by letting $\eta$ denote the probability that a pet reaches old age without developing behavioral problems and assuming that pets that develop such problems are euthanized with no psychic cost on the part of owners.

Fourth, by assuming all pets without owners are euthanized, we rule out the possibility of a feral population. This assumption is reasonable for dogs since feral dogs are dangerous and therefore typically eliminated by animal control. ${ }^{16}$ However, in many communities, there appear to be a significant population of feral cats. ${ }^{17}$ Recognizing the existence of a feral population would lead to an additional source of pet supply that could not be controlled via financial instruments such as taxes and subsidies.

Finally, in our modelling of the costs of euthanization, we ignore psychic costs bourne by nonowners who are nonetheless sympathetic to the plight of pets. Such costs are undoubtedly real because many citizens donate money and time to animal welfare organizations. ${ }^{18}$ However, the extent of the pet overpopulation problem suggests that such citizens form a small minority of the population. Relatedly, our measure of welfare ignores the costs of premature death to the animals themselves. This is because of difficulties in knowing how to think about these costs. Given the fixed number of potential owners, the premature death of one pet creates a home for another and it is not clear how to evaluate this trade off. Stated simply, would pets behind the veil of ignorance prefer a world in which they had a lower probability of being born and a longer life if born?

\section{Market equilibrium}

In a market equilibrium, pet allocation and production decisions are guided by the price mechanism and individual optimization. There are, in principle, markets for each of the four types of pets

\footnotetext{
${ }^{16}$ Dangers to the community include the transmission of rabies, dog bites, damage to livestock, and car accidents.

${ }^{17}$ For example, Patronek, Beck and Glickman's (1997) survey of pet owners in St Joseph County, Indiana revealed that $24 \%$ reported feeding free-roaming cats that they did not own.

18 According to survey evidence, 17 percent of households, including both owners and non-owners, reported monetary donations to animal causes, with an average donation among donors of $\$ 76$ per year (Frank, 2001). In addition, 10 percent reported volunteering their time, with an average of 54 hours per year among volunteers.
} 
(young pure breeds, young mixed breeds, old pure breeds, and old mixed breeds) and prices are determined by the usual requirement that demand equal supply. However, given the limited number of owners, there may be excess supply for some pet types even at a zero price. In this case, owners relinquish such pets to a state-run shelter and owners looking for pets of this type go to the shelter where they can obtain them free of charge. Surplus pets are euthanized by the shelter, which is financed by a head tax. Let $q_{a i t}$ denote the price of a pet of age $a$ and breed $i$ in period $t$ and let $\mathbf{q}_{t}$ denote the vector of period $t$ prices $\left(q_{y p t}, q_{y m t}, q_{o p t}, q_{o m t}\right)$. Similarly, let $\pi_{a i t}$ denote the probability that a pet of age $a$ and breed $i$ taken to the shelter in period $t$ will be destroyed and let $\boldsymbol{\pi}_{t}$ denote the vector of period $t$ euthanizing probabilities. Obviously, if $q_{a i t}>0$, then $\pi_{a i t}=0$.

Given the first period populations of old and young pets $\left(\left(\mathbf{H}_{1}^{o}, \mathbf{L}_{1}^{o}\right)\right.$ and $\left.\mathbf{y}_{1}\right)$ and the transition equation $\left(\mathbf{H}_{t+1}^{o}, \mathbf{L}_{t+1}^{o}\right)=\eta\left(\mathbf{H}_{y t}^{n}, \mathbf{L}_{y t}^{n}\right)$, a feasible allocation and production plan $\left(\mathbf{H}_{t}^{n}, \mathbf{L}_{t}^{n}, \mathbf{y}_{t+1}\right)_{t=1}^{\infty}$ is a market equilibrium if there exist prices $\left(\mathbf{q}_{t}\right)_{t=1}^{\infty}$ and euthanization probabilities $\left(\boldsymbol{\pi}_{t}\right)_{t=1}^{\infty}$ such that in each period $t$ four conditions are satisfied. First, owners choose to breed and spay their animals so as to generate the population of young pets in period $t+1, \mathbf{y}_{t+1}$. Second, new owners choose pets in a way consistent with $\left(\mathbf{H}_{t}^{n}, \mathbf{L}_{t}^{n}\right)$. Third, for any pet type whose price is positive, demand equals supply. Fourth, for any pet type with a zero price, supply exceeds demand and the actual euthanization probabilities are equal to $\boldsymbol{\pi}_{t}$.

We maintain four assumptions in our analysis of market equilibrium. The first is that the expected cost associated with a litter is less than the cost of spaying.

\section{Assumption 1}

$$
\varphi c_{l} \leq c_{s}
$$

This assumption implies that if owners could find homes for their pets' mixed breed offspring, they would choose not to spay them. It therefore implies that young mixed breeds will be in excess supply. Easily relaxed, the assumption focuses the analysis on the main case of interest.

The second assumption is that the benefit of owning a young mixed breed is positive.

\section{Assumption 2}

$$
\beta-c_{s}+\delta \eta\left[\alpha \beta-(1-\alpha) \xi_{o}\right]>0
$$

The left hand side represents the discounted expected benefit from owning a young mixed breed, assuming that it is spayed and euthanized if the owner has to give it up. In the first period of the 
pet's life, the owner gets a payoff $\beta-c_{s}$. If the pet survives until the second period, an event with probability $\eta$, the owner can keep the pet with probability $\alpha$ and obtain a benefit $\beta$. If he has to give the pet up, he suffers a loss $\xi_{o}$.

The third assumption is that, for a high type, the additional benefit of owning a pure rather than mixed breed exceeds the associated production cost.

\section{Assumption 3}

$$
\theta(1+\delta \eta \alpha)>\frac{c_{l}+r-c_{s}}{\delta x}
$$

The left hand side represents the additional discounted expected benefit of owning a pure breed for a high type. In the first period of the pet's life, the owner gets an additional payoff $\theta$. If the pet survives until the second period, an event with probability $\eta$, the owner can keep the pet with probability $\alpha$ and obtain an additional payoff $\theta$. The right hand side represents the production costs of a pure breed. A litter of pure breeds creates a cost of $c_{l}+r$. On the other hand, it will be necessary to spay one less pure breed, saving spaying costs of $c_{s}$. The cost of an additional litter is therefore $c_{l}+r-c_{s}$ and dividing through by $x$ yields the per-pet cost.

The fourth assumption is that for both types of owners, the benefit from a young pet exceeds that from a pair of old shelter pets.

\section{Assumption 4(i)}

$$
\beta-c_{s}+\delta \eta\left[\alpha \beta-(1-\alpha) \xi_{o}\right]>\lambda \beta(1+\delta \eta \alpha)
$$

4(ii)

$$
\beta+\theta-c_{s}+\delta \eta\left[\alpha(\beta+\theta)-(1-\alpha) \xi_{o}\right]-\frac{c_{l}+r-c_{s}}{\delta x}>\lambda(\beta+\theta)(1+\delta \eta \alpha) .
$$

Inequality 4(i) is for low types. The left hand side represents the benefit of owning a young mixed breed and the right hand side the benefit of instead owning a pair of old mixed breeds. ${ }^{19}$ Inequality 4(ii) is for high types. The left hand side represents the benefit of a young pure breed (net of production cost) and the right hand side the benefit of a pair of old pure breeds.

Under these assumptions, there exists a market equilibrium in which in periods $t \geq 2$, high type new owners are allocated young pure breeds and low types young mixed breeds, so that $\mathbf{H}_{t}^{n}=\left(\gamma_{H}-\alpha H_{t}^{o}, 0,0,0\right)$ and $\mathbf{L}_{t}^{n}=\left(0, \gamma_{L}-\alpha L_{t}^{o}, 0,0\right)$. The production of young pure breeds is sufficient to meet demand in the next period so that $y_{p t}=\gamma_{H}-\alpha H_{t}^{o}$. The production of young

\footnotetext{
19 Obviously, Assumption 4(i) implies Assumption 2. Nonetheless, it is worth distinguishing them as they play different roles in the analysis.
} 
mixed breeds exceeds demand and is given by $y_{m t}=\left(\gamma_{L}-\alpha L_{t}^{o}\right) /\left[1-\left(c_{s}-\varphi c_{l}\right) / \varphi x \delta \xi_{y}\right]$. The equilibrium price of young pure breeds in periods $t \geq 2$ is given by

$$
q_{y p t}=q_{p} \equiv \frac{c_{l}+r-c_{s}}{\delta x}
$$

and the prices of young mixed breeds and old pets are zero, so that $\mathbf{q}_{t}=\left(q_{p}, 0,0,0\right)$. Relinquished old pets are euthanized with probability 1 and young mixed breeds taken to the shelter are euthanized with probability

$$
\pi_{y m t}=\pi_{m} \equiv \frac{c_{s}-\varphi c_{l}}{\varphi(\eta x+(1-\eta)(x-\alpha)) \delta \xi_{y}},
$$

so that $\boldsymbol{\pi}_{t}=\left(0, \pi_{m}, 1,1\right)$.

There are four conditions to check to verify that the allocation and production plan we have described is a market equilibrium. ${ }^{20}$ The first is that owners would choose to breed and spay their young pets so as to generate the specified supply. We show in the Appendix that given the price of young mixed breeds is zero and the euthanization probability is (11), mixed breed owners will be indifferent between spaying or not. Similarly, given the price (10), pure breed owners will be indifferent between breeding or spaying. This indifference means that the supply of young pets is perfectly elastic at the equilibrium prices and euthanization probabilities. The second condition is that new owners will choose pets in a way consistent with $\left(\mathbf{H}_{t}^{n}, \mathbf{L}_{t}^{n}\right)$. This requires that high types purchase young pure breeds and low types obtain young mixed breeds. The other options for high types are not to acquire a pet or to get a young mixed breed or an old pure breed from the shelter. For low types, the relevant alternatives are not to acquire a pet, to purchase a young pure breed, or to get an old shelter pet. The Appendix provides a detailed demonstration that the postulated equilibrium behavior is optimal, but it follows fairly directly from Assumptions 2-4. The third condition, that supply equals demand for any pet type whose price is positive, is satisfied by construction. The fourth condition, that supply exceeds demand for any pet type with a zero price and the actual euthanization probabilities are equal to $\boldsymbol{\pi}_{t}$, can also shown to be satisfied.

\footnotetext{
20 Notice that our description of the allocation and production plan is incomplete because we have not described the allocation of young and relinquished old pets among new owners in period 1. However, this only determines the number of old owners at the beginning of period 2 and our description of the allocation and production plan from this point on is conditional on this in that period 2 production is given by $\mathbf{y}_{2}=\left(\gamma_{H}-\alpha H_{2}^{o},\left(\gamma_{L}-\alpha L_{2}^{o}\right) /[1-\right.$ $\left.\left.\left(c_{s}-\varphi c_{l}\right) / \varphi x \delta \xi_{y}\right]\right)$. Thus, from the viewpoint of the description of the equilibrium, it does not really matter how young and relinquished old pets are allocated in period 1.
} 
The equilibrium implies a dynamic evolution of the population of pets. In period $t \geq 2$, the population of young pets is given by $\left(y_{p t}, y_{m t}\right)=\left(\gamma_{H}-\alpha H_{t}^{o},\left(\gamma_{L}-\alpha L_{t}^{o}\right) /\left(1-\left(c_{s}-\varphi c_{l}\right) / \delta \varphi x \xi_{y}\right)\right)$. Since young pure breeds are owned by high types and uneuthanized young mixed breeds by low types, it must be the case that if $t \geq 3,\left(H_{t}^{o}, L_{t}^{o}\right)=\left(\eta y_{p t-1}, \eta y_{m t-1}\left(1-\left(c_{s}-\varphi c_{l}\right) / \delta \varphi x \xi_{y}\right)\right)$. It follows that

$$
\left(y_{p t}, y_{m t}\right)=\left(\gamma_{H}-\alpha \eta y_{p t-1}, \frac{\gamma_{L}}{1-\left(c_{s}-\varphi c_{l}\right) / \delta \varphi x \xi_{y}}-\alpha \eta y_{m t-1}\right)
$$

Given these dynamics, it is easy to show that the pet populations converge to the steady state

$$
\left(y_{p}, y_{m}\right)=\left(\frac{\gamma_{H}}{1+\alpha \eta}, \frac{\gamma_{L}}{(1+\alpha \eta)\left(1-\left(c_{s}-\varphi c_{l}\right) / \delta \varphi x \xi_{y}\right)}\right) .
$$

Thus, we have:

Proposition 1 Suppose that Assumptions 1, 2, 3 and 4 are satisfied. Then, there exists a market equilibrium in which in period 2 and beyond, high type new owners receive young pure breeds and low type new owners receive young mixed breeds. All relinquished old pets are euthanized. The price of young pure breeds is positive, but young mixed breeds are in excess supply and some are euthanized. The population of young pets converges to the steady state (13).

The equilibrium described in Proposition 1 captures key qualitative features of the markets for dogs and cats in the U.S.. As noted in Section 2, for both dogs and cats, old pets relinquished by their owners are unlikely to be adopted at shelters. Pure breed puppies and kittens, on the other hand, are sold for significant prices. ${ }^{21}$ Mixed breed kittens are in excess supply and some are euthanized. The same is true for mixed breed puppies in many communities. ${ }^{22}$

\section{$5 \quad$ Pet overpopulation}

To provide a benchmark with which to compare the plan generated by the market, we now study efficient plans. A plan is efficient if it is feasible and there is no other feasible plan which generates a higher level of lifetime surplus. The efficiency problem is challenging, with many different decisions

\footnotetext{
${ }^{21}$ As noted in Section 2, the market for pure breed dogs is much larger than that for pure breed cats, suggesting that the fraction of the population who have a preference for pure breeds (i.e., high types) is much greater for potential dog owners.

22 If Assumption 1 did not hold so that the expected cost of a litter exceeded the cost of spaying, the price of young mixed breed pets would have to be positive in equilibrium and none would be euthanized. Thus, the model suggests that communities in which mixed breed puppies are sold for a positive price will be those in which the expected costs of a litter are low relative to the cost of spaying.
} 
to be determined and a complex set of feasibility constraints. However, given that the market equilibrium converges to a steady state, we can simplify matters by assuming that the efficient plan converges to a steady state. Comparing this with the equilibrium steady state will allow us to draw conclusions concerning market efficiency.

We impose two additional assumptions. The first is that when all a pet's offspring are euthanized, the expected social cost of it having a litter exceeds the cost of spaying.

\section{Assumption 5}

$$
\varphi\left[\delta x\left(\xi_{y}+\rho\right)+c_{l}\right]>c_{s} .
$$

The left hand side is the expected social cost of a young pet having a litter under the assumption that all its offspring are euthanized.

The second assumption is that for both types of owners, the social benefits from young pets are less than those from a pair of old shelter pets.

\section{Assumption 6(i)}

$$
\beta-c_{s}+\delta \eta\left[\alpha \beta-(1-\alpha) \xi_{o}\right]+\frac{c_{s} / \varphi-c_{l}}{\delta x}<\left(\lambda \beta+\xi_{o}+\rho\right)(1+\delta \eta \alpha) .
$$

6(ii)

$$
\beta+\theta-c_{s}+\delta \eta\left[\alpha(\beta+\theta)-(1-\alpha) \xi_{o}\right]-\frac{c_{l}+r-c_{s}}{\delta x}<\left[\lambda(\beta+\theta)+\xi_{o}+\rho\right](1+\delta \eta \alpha) .
$$

This assumption should be contrasted with Assumption 4, which concerned private benefits. The key difference is the inclusion of the euthanizing costs $\xi_{o}+\rho$. Assumption 6 recognizes that if an owner owns two old pets instead of a young pet, then the old pets are spared from being euthanized and this saves society $\xi_{o}+\rho$ per pet. Assumption 6(i) also recognizes that it is socially costly to prevent the production of young mixed breeds since the production cost is negative under Assumption 1.

With these Assumptions understood, we now present our characterization of the efficient steady state.

Proposition 2 Suppose that Assumptions 1, 2, 3, 5 and 6 are satisfied and that the efficient plan converges to a steady state. Then, in this steady state, no pets are euthanized. If

$$
\theta(1+\delta \eta \alpha)(1-\lambda)>\frac{r+c_{s}(1-\varphi) / \varphi}{\delta x},
$$


high type new owners receive young pure breeds and low type new owners receive young mixed breeds or relinquished old pets. The population of young pets is

$$
\left(y_{p}, y_{m}\right)=\left(\frac{\gamma_{H}}{1+\alpha \eta}, \frac{\gamma_{L}(1+\alpha \eta)-(1-\alpha) \eta \gamma_{H}}{(1+\eta)(1+\alpha \eta)}\right) .
$$

If inequality (14) is not satisfied, high type new owners receive young or relinquished old pure breeds and low type new owners receive young or relinquished old mixed breeds. The population of young pets is

$$
\left(y_{p}, y_{m}\right)=\left(\frac{\gamma_{H}}{1+\eta}, \frac{\gamma_{L}}{1+\eta}\right) .
$$

The most notable feature of the efficient steady state is that no pets are euthanized. Not only are no surplus young pets produced, but also all relinquished old pets are allocated to new owners. This reflects Assumptions 5 and 6 which bound below the costs of euthanizing young and old pets. Together these assumptions imply that a "no kill" policy is optimal in the steady state. ${ }^{23}$

A further interesting feature is the way relinquished old pets are allocated among new owners. If high types have a sufficiently strong preference for pure breeds so that (14) is satisfied, all relinquished old pets are allocated to low types. The additional benefit high types receive from owning young pure breeds is sufficiently large that it is inefficient to deny them ownership. If high types have only a weak preference, relinquished old pure breeds are allocated to high types and mixed breeds to low types. In the latter case, the steady state population of young pure breeds is lower than in the former as can be seen by comparing (15) and (16).

Comparing Proposition 2 with our analysis of the market, we see that when Assumptions 1 through 6 are satisfied, the market equilibrium described in Proposition 1 is inefficient. The inefficiency arises because pets are euthanized in equilibrium. The result is that the equilibrium steady state population of pets exceeds the efficient steady state level. In this sense, therefore, the equilibrium exhibits pet overpopulation.

The extent of pet overpopulation can be calculated by subtracting the efficient population of young pets from the equilibrium population. From (15) and (16), the steady state efficient

\footnotetext{
${ }^{23}$ As discussed in Section 3.4, the analysis ignores the problem of pets relinquished for behavioral problems. It is clear that such animals will need to be euthanized and hence a "no-kill" policy can only apply to adoptable pets.
} 
population of young pets is $1 /(1+\eta)$. Thus, from $(13)$, the degree of pet overpopulation is

$$
\frac{1}{1+\alpha \eta}\left[\gamma_{H}+\frac{\gamma_{L}}{1-\left(c_{s}-\varphi c_{l}\right) / \delta \varphi x \xi_{y}}\right]-\frac{1}{1+\eta} .
$$

This difference is decreasing in $\alpha, \varphi, c_{l}, \xi_{y}, x$ and $\delta$, and increasing in $\eta, \gamma_{L}$ and $c_{s}$. Higher spaying costs, lower litter costs, and lower owner psychic costs will therefore increase overpopulation.

The inefficiency of the market equilibrium reflects what is essentially a common pool externality problem. The "common pool" is the limited number of homes available for pets. When owners of young pets make their breeding or spaying decisions, they do not take into account that their offspring will crowd out homes for other pets. This crowding out is costly because euthanizing animals is costly. The cost stems from two sources: the direct resource cost and, more importantly, the fact that owners care about the fate of the animals that they must relinquish.

It is well known that externalities stem from missing markets. ${ }^{24}$ In our application, the inefficiency would be eliminated: if (i) owners whose pets were euthanized were charged a user fee by the shelter equal to the resource $\operatorname{cost} \rho$, and, (ii) there were an "adoption market" in which owners with surplus pets could pay new owners to adopt them. Charging for the resource cost would ensure that owners fully internalized the cost of euthanizing their pets and would reduce the relative payoff of owning young pets. Opening an adoption market would ensure that efficient trades between new owners and owners with surplus pets were realized. The market would involve relinquishing old owners paying new owners to adopt their pets rather than obtaining young pets. In response, the production of young pets would be scaled back and euthanization reduced. The market would also involve owners of mixed breed puppies and kittens paying new owners to adopt their pets. These payments would raise the cost of pets having litters and thereby increase spaying.

Both parts of this market fix have obvious practical difficulties. The difficulty with shelters charging user fees is that relinquishing owners could avoid them either by dumping their pets in the wild, or by claiming they were bringing in strays. The difficulty with the adoption market is that unscrupulous individuals could claim to adopt a surplus pet, pick up the payment, and then dump it in the wild. This said, elements of this market solution can be identified in the real world. It is commonplace, for example, for owners to persuade friends and relatives to take on their surplus pets by offering to pay for food and veterinary expenses. In these cases, the personal connection limits the agency problem. However, the scarcity of personal connections also limits

${ }^{24}$ See, for example, Mas-Colell, Whinston, and Green (1995). 
the reach of this solution.

\section{Combating pet overpopulation}

The previous two sections identify assumptions under which the market for pets is inefficient. The inefficiency arises because pets are euthanized in equilibrium and this results in the population of young pets exceeding the efficient steady state level. Since the problem stems from a common pool externality, it is clear that an appropriate set of Pigouvian taxes and subsidies will improve efficiency. But the question is exactly what type of corrective policies will be helpful? This section addresses this question.

Given the objective of identifying corrective policies that can be imposed on the market, we assume that pet populations at the time of intervention are consistent with those arising in market equilibrium. Accordingly, we suppose that period 1 is the first period of intervention and make

\section{Assumption 7}

$$
H_{m 1}^{o}=L_{p 1}^{o}=0, y_{p 1}=\gamma_{H}-\alpha H_{1}^{o} \text {, and } y_{m 1} \geq \gamma_{L}-\alpha L_{1}^{o} \text {. }
$$

Thus, high type old owners own pure breeds and low types mixed breeds. Moreover, the population of young pure breeds equals the number of high type new owners and the population of young mixed breeds exceeds the number of low type new owners. Given Proposition 1, this is consistent with intervention taking place after the market has been guiding allocation and production for two or more periods.

The optimal corrective policies depend on the strength of high types' preference for pure breeds. There are two cases, depending on whether or not (14) holds.

\subsection{Strong preferences for pure breeds}

If (14) holds, in period 1 and beyond, impose a spaying subsidy equal to

$$
s_{s}=c_{s}-\varphi c_{l},
$$

and, in period 2 and beyond, a tax on young pets equal to

$$
T_{y}=\beta(1-\lambda)(1+\delta \eta \alpha)-\varphi c_{l} .
$$


Under these policies, there exists a market equilibrium in which from period 2 onwards, high type new owners are allocated young pure breeds and low types either young mixed breeds or relinquished old pets. This means that $\mathbf{H}_{t}^{n}=\left(\gamma_{H}-\alpha H_{t}^{o}, 0,0,0\right)$ and $\mathbf{L}_{t}^{n}=\left(0, \gamma_{L}-L_{t}^{o}-(1-\right.$ $\left.\alpha) H_{t}^{o},(1-\alpha) H_{t}^{o},(1-\alpha) L_{t}^{o}\right)$. The production of young pets is sufficient to meet demand in the next period so that $\mathbf{y}_{t}=\left(\gamma_{H}-\alpha H_{t}^{o}, \gamma_{L}-L_{t}^{o}-(1-\alpha) H_{t}^{o}\right)$. The producer price of young pure breeds in periods $t \geq 2$ supporting this equilibrium is

$$
q_{y p t} \equiv q_{y p}=\frac{(1-\varphi) c_{l}+r}{\delta x} .
$$

The prices of young mixed breeds and old pets are zero, as are the probabilities that all pets are euthanized.

There are three key steps in establishing that this is an equilibrium. First, we must show that owners will choose to breed and spay their animals so as to generate the specified supply. This is established by noting that the subsidy (18) makes mixed breed owners indifferent between spaying or not, and the price (20) makes pure breed owners indifferent between breeding or spaying. Second, we must demonstrate that with the tax (19), low types must be indifferent between obtaining young mixed breeds or relinquished old pets. Third, we must show high types prefer purchasing young pure breeds to getting old pure breeds from the shelter. This step uses the fact that (14) is satisfied.

Turning to dynamics, in period $t \geq 2$, the population of young pets is given by $\left(y_{p t}, y_{m t}\right)=$ $\left(\gamma_{H}-\alpha H_{t}^{o}, \gamma_{L}-L_{t}^{o}-(1-\alpha) H_{t}^{o}\right)$. Since young pure breeds are owned by high types and young mixed breeds by low types, if $t \geq 3,\left(H_{t}^{o}, L_{t}^{o}\right)=\left(\eta y_{p t-1}, \eta y_{m t-1}\right)$. It follows that

$$
\left(y_{p t}, y_{m t}\right)=\left(\gamma_{H}-\alpha \eta y_{p t-1}, \gamma_{L}-\eta y_{m t-1}-(1-\alpha) \eta y_{p t-1}\right)
$$

Given these dynamics, the pet populations converge to the steady state (15).

The fact that the equilibrium converges to the efficient steady state does not imply that it generates higher lifetime surplus than the uncorrected market equilibrium. Short run losses in the transition could overwhelm long run gains. Fortunately, however, we can prove directly that the corrected equilibrium generates higher lifetime surplus. Thus, we have:

Proposition 3 Suppose that Assumptions 1-7 are satisfied and that (14) holds. Suppose further that the spaying subsidy (18) is offered in period 1 and beyond, and that the tax (19) is imposed on owners of young pets in period 2 and beyond. Then, there exists a market equilibrium under 
these policies in which in period 2 and beyond, no pets are euthanized. High type new owners receive young pure breeds and low type new owners receive young mixed breeds or relinquished old pets. The population of young pets converges to the efficient steady state (15) and the equilibrium generates higher lifetime surplus than the unregulated equilibrium described in Proposition 1.

\subsection{Weak preferences for pure breeds}

If (14) does not hold, differential taxes on young pure and mixed breeds are required. Specifically, in period 1 and beyond, introduce a spaying subsidy equal to (18) as before, and, in period 2 and beyond, impose a tax on owners of young mixed breeds equal to

$$
T_{y m}=\beta(1-\lambda)(1+\delta \eta \alpha)-\varphi c_{l},
$$

and a tax on owners of young pure breeds equal to

$$
T_{y p}=(\beta+\theta)(1-\lambda)(1+\delta \eta \alpha)-\left(\frac{(1-\varphi) c_{l}+r}{\delta x}\right)-\varphi c_{l} .
$$

Under these policies, there exists a market equilibrium in which from period 2 onwards, high type new owners are allocated young or relinquished old pure breeds and low types young or relinquished old mixed breeds. This means that $\mathbf{H}_{t}^{n}=\left(\gamma_{H}-H_{t}^{o}, 0,(1-\alpha) H_{t}^{o}, 0\right)$ and $\mathbf{L}_{t}^{n}=$ $\left(0, \gamma_{L}-L_{t}^{o}, 0,(1-\alpha) L_{t}^{o}\right)$. The production of young pets is sufficient to meet demand in the next period so that $\mathbf{y}_{t}=\left(\gamma_{H}-H_{t}^{o}, \gamma_{L}-L_{t}^{o}\right)$. The producer price of young pure breeds in periods $t \geq 2$ remains given by (20). The prices of young mixed breed and old pets are zero, as are the probabilities that all pets are euthanized.

The only new step in showing this is an equilibrium is demonstrating that, given the tax (23), high types are indifferent between young and relinquished old pure breeds. An interesting fact about the equilibrium is that the corrective tax on pure breeds may be lower than that on mixed breeds. From (22) and (23), we see that

$$
T_{y m}-T_{y p}=\left(\frac{(1-\varphi) c_{l}+r}{\delta x}\right)-\theta(1-\lambda)(1+\delta \eta \alpha) .
$$

Since (14) does not hold, the right hand side will be positive for $\varphi c_{l}$ sufficiently close to $c_{s}$. Despite the higher tax, low types are not tempted to purchase pure breeds because their total purchase price, $q_{y p}+T_{y p}$, exceeds that of mixed breeds, $T_{y m}$.

The dynamic evolution of this equilibrium is such that, in period $t \geq 2$, the population of young pets is given by $\left(y_{p t}, y_{m t}\right)=\left(\gamma_{H}-H_{t}^{o}, \gamma_{L}-L_{t}^{o}\right)$. Since young pure breeds are owned by 
high types and young mixed breeds by low types, if $t \geq 3,\left(H_{t}^{o}, L_{t}^{o}\right)=\left(\eta y_{p t-1}, \eta y_{m t-1}\right)$. It follows that

$$
\left(y_{p t}, y_{m t}\right)=\left(\gamma_{H}-\eta y_{p t-1}, \gamma_{L}-\eta y_{m t-1}\right) .
$$

Given these dynamics, the pet populations converge to the steady state (16).

We can again show that lifetime surplus is higher in this equilibrium than in the uncorrected market equilibrium and thus we have:

Proposition 4 Suppose that Assumptions 1-7 are satisfied and that (14) does not hold. Suppose further that the spaying subsidy (18) is offered in period 1 and beyond, and that the taxes (22) and (23) are imposed on owners of young pets in period 2 and beyond. Then, there exists a market equilibrium under these policies in which in period 2 and beyond, no pets are euthanized. High type new owners receive young or relinquished old pure breeds and low type new owners receive young or relinquished old mixed breeds. The population of young pets converges to the efficient steady state (16) and the equilibrium generates higher lifetime surplus than the unregulated equilibrium described in Proposition 1.

\subsection{Discussion}

Propositions 3 and 4 describe corrective policies that improve welfare and generate efficient pet populations in the long run. There are two key policy tools: taxes on the ownership of young animals and spaying subsidies. The taxes remedy the inefficiency resulting from the euthanization of relinquished old pets by shifting demand from young to old animals. The spaying subsidy remedies the inefficiency resulting from euthanization of young mixed breeds by choking off their supply.

When the efficient solution involves high type new owners receiving only young pure breeds, a uniform tax on young pets is all that is necessary. For when this tax is set at the level which makes low types indifferent between young mixed breeds and relinquished old pets, high types strictly prefer buying young pure breeds. When the optimum involves high types receiving both young and relinquished old pure breeds, differential taxes are necessary. Pure breeds may be taxed at a lower rate, which is perhaps counter-intuitive. The result reflects the fact that with a uniform tax sufficient to make low types indifferent, high types will strictly prefer relinquished old pets. A lower tax, therefore, is necessary to induce them to also purchase young pure breeds. 
How could these policies be implemented? As noted in Section 2, most localities require dog owners to purchase an annual license. Moreover, in many communities, license fees are already higher for intact dogs, which acts as a spaying subsidy. The policies could be implemented by making license fees also vary with a dog's age. This would only require that localities keep track of the ages of dogs in their communities. Implementing the policies for cats is more difficult because in most communities they are not licensed. In addition, it would seem harder to monitor both cat populations and ages.

\section{$7 \quad$ A calibrated example}

This section develops a calibrated example of the model. We choose parameters so that the properties of the equilibrium match the corresponding properties of the market for dogs in the U.S.. Using these parameters, we calculate the combination of taxes and subsidies required to implement the efficient steady state. We also quantify the welfare gains associated with a movement from the unregulated market equilibrium to the equilibrium under these optimal policies. Finally, we discuss results using alternative parameter values.

Before discussing the details, we first address a key simplification of the model. In particular, the model assumes a two-period lifetime for pets whereas dogs have a typical lifespan of around 10 years. Given this discrepancy, we interpret a period of the model as 5 years of elapsed time. We thus consider dogs between 0 and 5 as young and those between 6 and 10 as old.

The first panel of Table 1 provides a summary of our assumptions. There are three groups of parameters. The first, which consists of $\left\{\alpha, \eta, c_{s}, \rho, \varphi, \delta\right\}$, can be inferred directly from survey data, scientific studies, and related sources. Given that an estimated 15 percent of dogs are brought to shelters on an annual basis and that an estimated 25 percent of these relinquishments are due to changes in owner circumstances, we estimate the probability of future ownership $(\alpha)$ to be $0.81 .^{25}$ Based upon mortality data from the Veterinary Medical Data Base, Patronek, Waters, and Glickman (1997) estimate that around 25 percent of dogs have died by age 5.5, and we thus

\footnotetext{
25 According to APPMA (2005), there are around 74 million dogs in the U.S., and, according to the National Shelter Survey, 11 million, or 15 percent, are brought to shelters in any given year. According to Frank (2001), around 30 percent of these relinquishments are due to behavioral reasons. In addition, according to New et. al. (2000), around 45 percent of relinquished dogs are puppies, defined as those below age 1. Under the assumption that no puppies are relinquished for behavioral reasons, this implies that around 25 percent of relinquishments can be considered as likely due to changes in owner circumstance, and we thus estimate that 3.8 percent of dogs are brought to shelters on an annual basis for such reasons. This translates into a 19 percent rate over a 5 -year period.
} 
use 75 percent as an estimate of the probability of natural survival to old age. As mentioned in Section 3.4, however, we interpret the parameter $\eta$ as also incorporating relinquishment due to behavioral reasons, which we estimate to be 23 percent. ${ }^{26}$ Taken together, the 75 percent natural survival rate and the 77 percent of dogs without behavioral problems suggests a value for $\eta$ of 0.58 . According to Frank (2001), the cost of neutering or spaying a dog at a low cost clinic is around $\$ 100$, and we use this as our value for $c_{s} \cdot{ }^{27}$ Based upon information regarding the costs of euthanasia procedures and disposal, we set the resource cost of euthanasia ( $\rho$ ) equal to $\$ 100 .^{28}$ Household survey data on unplanned litters suggests that the probability of producing a mixed breed offspring $(\varphi)$ is equal to 21 percent. $^{29}$ Finally, using the 5 year length of a period and an assumed interest rate of 3 percent, we set the discount factor $(\delta)$ equal to 0.86 .

The second group of parameters, which consists of $\left\{\gamma_{H}, \gamma_{L}, x, c_{l}, r\right\}$, are those that can be inferred by matching key features of the market for dogs to their theoretical counterparts. We can infer the mix of high and low types directly from the distribution of pure and mixed breeds. This is because, in equilibrium, high types own pure breeds and low types mixed breeds. According to APPMA (2005), approximately 58 percent of dogs are pure-breeds, and we thus set $\gamma_{H}=0.58$ and $\gamma_{L}=0.42$.

We can calculate the size of a litter $(x)$ by comparing breeding rates to those implied by our model. Using the fact that breeding rates for young pure breeds in steady state must equal $1 / x$ and that the fraction of pure breeds that are young in the model is given by $1 /(1+\alpha \eta)$, we have that the equilibrium probability of breeding among all pure breeds is $1 / x(1+\alpha \eta)$. The household survey conducted by New et. al. (2000 and 2004) suggests that about 12 percent of owners choose

\footnotetext{
${ }^{26}$ Given that 15 percent of dogs are brought to shelters in any given year and that, according to Frank (2001), around 30 percent of these relinquishments are due to behavioral reasons, we estimate that around 4.5 percent of dogs are brought to shelters on an annual basis for such reasons. This implies a 23 percent relinquishment rate due to behavioral reasons over a 5 -year period for young dogs.

27 Note that this estimate does not include any psychic costs for owners from the spaying and neutering procedures. Many owners cite a belief that dogs should not be altered as a reason for leaving their dog intact, and this belief may increase the cost of spaying and neutering (Patronek, Beck, and Glickman, 1997). On the other hand, our estimate of $\$ 100$ may overstate the true cost since, as noted in Section 2, many municipalities have lower license fees for altered dogs.

28 Prices typically charged by vets for the euthanasia procedure and cremation total $\$ 135$. Since shelters may be more efficient and since some of the price charged by vets may include profits, we discount these figures somewhat when estimating shelter costs. These data are taken from the website http://www.dogbreedinfo.com/timetoletgo.htm (accessed June 2009).

29 According to New et. al. (2004), 1.4 percent of households had an unplanned litter in 1996. This translates into a 7 percent rate over a 5 -year period. Given that 60 percent of dogs in the survey are neutered and that 6 percent had planned litters over a 5-year period, 34 percent of dogs in the survey are neither neutered nor bred, and this suggests that $\varphi=0.07 / 0.34=0.21$.
} 
to breed their dogs. ${ }^{30}$ Using this and our previously assigned values of $\alpha$ and $\eta$, we thus estimate $x$ to be 5.67 .

Given a value for $\xi_{y}$, we can infer the cost of a litter $\left(c_{l}\right)$ and breeding costs $(r)$ from the equilibrium euthanasia probability (11) and price of pure breeds (10). Using our estimates of $\left\{\alpha, \eta, c_{s}, \varphi, \delta, x\right\}$ and an estimate of $\pi_{m}$, the cost of a litter can be obtained by inverting (11) as follows:

$$
c_{l}=\frac{c_{s}-\pi_{m} \varphi(\eta x+(1-\eta)(x-\alpha)) \delta \xi_{y}}{\varphi} .
$$

With this value for $c_{l}$ and an estimate of $q_{p}$, we can then invert the pricing equation (10) to solve for breeding costs $\left(r=\delta x q_{p}-c_{l}+c_{s}\right)$. The estimate we use of the euthanasia rate for young mixed breeds is 31 percent and that of the price of pure breed puppies is $\$ 489 .{ }^{31}$ We discuss how we set $\xi_{y}$ below.

The third group of parameters, which consist of $\left\{\beta, \theta, \lambda, \xi_{y}, \xi_{o}\right\}$, are those for which we have little information other than the constraints imposed by Assumptions 1-6. To address this issue, we present estimates for a variety of values. For the baseline analysis, we set the benefit from owning a young pet $(\beta)$ equal to 2000 and the benefit for high types from owning a pure breed $(\theta)$ to 2000 for the strong preference case and 1000 for the weak case. These should be interpreted as the 5 year flow of ownership benefits net of costs such as food and veterinary care. In addition, we set the deflation parameter $(\lambda)$ to 0.75 . We next set the value of the psychic costs associated with euthanizing young dogs $\left(\xi_{y}\right)$ to 100 . This implies that an owner delivering a litter of five puppies to a shelter incurs a psychic cost of 500 if he knows for certain that they will be euthanized. With this estimate of psychic costs in hand, we then calculate $c_{l}=334$ and $r=2151$ using the procedure in the preceding paragraph. Finally, for the euthanasia cost for old dogs, Assumption 6 provides a lower bound, which is equal to 444, and Assumption 4 provides an upper bound, which

\footnotetext{
${ }^{30}$ In their household survey, 1.2 percent of households report a planned litter in 1996 and about one-half of dogs in their survey are pure breeds. Assuming that all planned deliveries are by pure breeds, as is suggested by our model, then 2.4 percent of pure breeds had a planned litter. Over a 5-year period, this suggests a breeding rate of 12 percent.

31 The estimate of the price of pure breed puppies is taken from APPMA (2005). To understand the estimate of the euthanasia probability, first note that we can write $\operatorname{Pr}($ euthanasia $)=\operatorname{Pr}($ euthanasia $\mid$ young $) \operatorname{Pr}($ young $)+$ $\operatorname{Pr}($ euthanasia $\mid$ old $) \operatorname{Pr}($ old $)$. In our model we have that $\operatorname{Pr}($ euthanasia|old $)=1$, so we can write $\operatorname{Pr}($ euthanasia $\mid$ young $)$ $=[\operatorname{Pr}($ euthanasia $)-\operatorname{Pr}($ old $)] / \operatorname{Pr}$ (young). We use estimates of $\operatorname{Pr}($ euthanasia $)=0.69$ from the National Shelter Survey. To calculate the fraction of dogs brought to shelters who are young, we use information from New et. al. (2000) on the age distribution of dogs. For the purposes of this calculation, we define a young dog to be less than one year of age and thus implicitly assume that all dogs between ages 1 and 5 have behavioral problems and are thus not adoptable. Given that 45 percent of dogs relinquished to shelters are below one year of age, we estimate $\operatorname{Pr}($ euthanasia $\mid$ young $)=0.31$.
} 
is 4900 . As a baseline value, we set $\xi_{o}$ equal to a relatively conservative 800 .

Given these assumed parameters, we now use the model to conduct a numerical welfare analysis. $^{32}$ Starting with the strong preference case, the optimal tax on young dogs, as shown in the second panel of Table 1, equals $\$ 632$. This is substantial, given that it exceeds current prices of pure breed puppies. Introducing a tax of this magnitude would represent a dramatic change in policy and would raise significant revenues. In the weak preference case, the tax on mixed breeds remains at $\$ 632$ but the tax on young pure breeds is a substantially lower $\$ 488$. This illustrates the point made in the previous section concerning the relative size of taxes on mixed and pure breeds. In both cases, the required subsidy on spaying equals $\$ 30$.

We next compute the welfare gains associated with introducing these optimal policies. In particular, we assume the initial populations of old and young pets are those associated with the equilibrium steady state (16) and calculate the difference in lifetime surplus between the corrected and uncorrected equilibrium. ${ }^{33}$ These surplus differences are denominated in dollars and, after dividing by the size of the population $(1 / \alpha)$, can be interpreted as the increase in discounted lifetime surplus for an average potential owner. As shown in the final panel of Table 1 , we calculate average welfare gains of $\$ 239$ in the first case and $\$ 185$ in the second. To obtain the aggregate welfare gain, we need to multiply these per capita gains by the number of potential owners. One way to estimate the number of potential owners would be to note that there were 106 million households in the U.S. in 2000 and that dog ownership rates are 39 percent, which would imply that the number of potential owners is 51 million. However, this neglects the fact that, in contrast to the model, many households own multiple dogs. Recognizing this, we count a household owning $n$ dogs as $n$ potential owners. Given that, as noted in Section 2, owners have 1.7 dogs on average, this leads to an estimate of the number of potential owners of 87 million. The aggregate welfare gain is therefore approximately $\$ 21$ billion in the first case and $\$ 16$ billion in the second.

Of course, these baseline results are contingent upon our assumed parameter values. To provide a feel for sensitivity, we next present results for four alternative scenarios. As shown in Table 2, we first increase by 25 percent, relative to their baseline values, the benefit from owning a young pet $(\beta)$ and the benefit for high types from owning a pure breed $(\theta)$. As shown, this increases the

\footnotetext{
32 We have verified that Assumptions 1-6 are satisfied under this set of parameter values.

33 We make use of expressions for the welfare gains that are developed in the proofs of Propositions 3 and 4 .
} 
required taxes since the prices of young pets must be even higher to induce owners to adopt older pets. The welfare gains are similar in magnitude to their baseline values. We next decrease $\beta$ and $\theta$ by 25 percent, relative to their baseline values. As shown, this decreases the required taxes, but, again, the welfare gains are similar in magnitude to their baseline values. In the third scenario, we return $\beta$ and $\theta$ to their baseline values and instead increase the psychic costs ( $\xi_{y}$ and $\left.\xi_{o}\right)$ from euthanasia by 25 percent, relative to their baseline values. As shown, the required taxes and subsidies change only slightly in this case. The welfare gains, by contrast, are significantly higher. This reflects the fact that these higher psychic costs are avoided in the corrected equilibrium. Finally, we consider a scenario in which pyschic costs are reduced by 25 percent from their baseline values. As expected, the welfare gains are smaller in this case.

\section{Conclusion}

Pet overpopulation is a theoretically interesting and practically relevant economic problem which has not received much attention from economists. The economic model developed in this paper provides a clean way of thinking about the problem and sheds light on how to correct it. Moreover, the quantitative analysis based on the model suggests that there may be significant welfare gains to such a correction.

While we feel the paper makes significant progress, there are limitations that could be addressed in future work. Incorporating gender and considering the relative effectiveness of spaying versus neutering would be interesting. So too would be moving beyond two-period lived pets and shedding more light on how taxes should depend on age. Finally, it would be useful to relax the assumption of a perfectly inelastic demand for pets by introducing more heterogeneity in ownership benefits. This would permit consideration of aggregate demand responses to corrective policies. 


\section{References}

Allen, R.C. and I. Keay, (2004), "Saving the Whales: Lessons from the Extinction of the Eastern Arctic Bowhead," Journal of Economic History, 64(2), 400-432.

American Veterinary Medical Association, (2002), U.S. Pet Ownership and Demographic Sourcebook.

American Pet Products Manufacturer Association, (2005), National Pet Owners Survey.

Carlos, A. and F. Lewis, (1993), "Indians, the Beaver, and the Bay: The Economics of Depletion in the Lands of the Hudson Bay Company," Journal of Economic History, 53(3), 465-494.

DiGiacomo, N., Arluke, A. and G. Patronek, (1998), "Surrendering Pets to Shelters: The Relinquisher's Perspective," Anthrozoos, 11(1), 41-51.

Endenburg, N., 't Hart, H. and J. Bouw, (1994), "Motives for Acquiring Companion Animals," Journal of Economic Psychology, 15(1), 191-206.

Frank, J., (2001), A New Economic Paradigm for Addressing Dog Overpopulation and the Effectiveness of Policy Alternatives, Doctoral dissertation, Rensselaer Polytechnic Institute, Troy, NY.

Frank, J., (2004), "An Interactive Model of Human and Companion Animal Dynamics: The Ecology and Economics of Dog Overpopulation and the Human Costs of Addressing the Problem," Human Ecology, 32(1), 107-129.

Gordon, H. S., (1954), "Economic Theory of a Common Property Resource: The Fishery," Journal of Political Economy, 62(1), 124-142.

Hirschman, E., (1994), "Consumers and their Animal Companions," Journal of Consumer Research, 20(4), 616-632.

Kremer, M. and C. Morcom, (2000), "Elephants," American Economic Review, 90(1), 212234 .

Lepper, M., Kass, P. and L. Hart, (2002), "Prediction of Adoption Versus Euthanasia Among Dogs and Cats in a California Animal Shelter," Journal of Applied Animal Welfare Science, $5(1), 29-42$.

Mas-Colell, A., Whinston, M. and J. Green, (1995), Microeconomic Theory, Oxford University Press: New York, NY.

New, J., Salman, M., King, M., Scarlett, J., Kass, P. and J. Hutchison, (2000), "Characteristics of Shelter-Relinquished Animals and their Owners Compared with Animals and their Owners in U.S. Pet-owning Households," Journal of Applied Animal Welfare Science, 3(3), 179-201.

New, J., Hutchinson, J., Salman, M., King, M., Scarlett, J. and P. Kass, (2004), "Birth and Death Rate Estimates for Cats and Dogs in U.S. Households and Related Factors," Journal of Applied Animal Welfare Science, 7(4), 229-241.

Patronek, G., Beck, A. and L. Glickman, (1997), "Dynamics of Dog and Cat Populations in a Community," Journal of the American Veterinary Medical Association, 210(5), 637-642. 
Patronek, G., Waters, D. and L. Glickman, (1997), "Comparative Longevity of Pet Dogs and Humans: Implications for Gerontology Research," Journals of Gerontology Series A: Biological and Medical Sciences, 52(3), 171-178.

Rosen, S., (1987), "Dynamic Animal Economics," American Journal of Agricultural Economics, 69(3), 547-557.

Rosen, S., Murphy, K. and J. Scheinkman, (1994), "Cattle Cycles," Journal of Political Economy, 102(3), 468-492.

Schwarz, P., Troyer, J. and J. Walker, (2007), "Animal House: Economics of Pets and the Household," The B.E. Journal of Economic Analysis \& Policy: Contributions, 7(1), article 35 .

Sydsaeter, K., (1981), Topics in Mathematical Analysis for Economists, Academic Press: New York, NY.

Taylor, M. S., (2007), "Buffalo Hunt: International Trade and the Virtual Extinction of the North American Bison," NBER Working Paper \# 12969. 


\section{$9 \quad$ Appendix $^{34}$}

\subsection{Proof of Proposition 1}

We begin by verifying that the allocation and production plan described in the text is a market equilibrium. The first condition to check is that owners would choose to breed and spay so as to generate the specified supply. This requires that in period $t-1,\left(\gamma_{H}-\alpha H_{t}^{o}\right) / x$ owners of young pure breeds will breed their pets and $\left(\gamma_{L}-\alpha L_{t}^{o}\right) /\left[\varphi x-\left(c_{s}-\varphi c_{l}\right) / \delta \xi_{y}\right]$ owners of young pets will neither spay nor breed. We claim that given the price of young mixed breeds is zero and that the euthanization probability is (11), owners of young mixed breeds will be indifferent between spaying or not. Consider the cost to such an owner of having a litter. In period $t-1$ he incurs a cost $c_{l}$. In period $t$, he incurs a further psychic cost of $x \pi_{m} \xi_{y}$ if the parent pet survives. For in this case, he will give up all the offspring. If the parent dies, the owner again loses $x \pi_{m} \xi_{y}$ if he cannot own a pet but only $(x-1) \pi_{m} \xi_{y}$ if he can because he can keep one of the litter. The probability $\pi_{m}$ is such that $c_{s}$ equals $\varphi\left[c_{l}+\delta(\eta x+(1-\eta)(x-\alpha)) \pi_{m} \xi_{y}\right]$, which makes owners indifferent between spaying or not. Similarly, we claim that given the price (10), owners of young pure breeds will be indifferent between breeding or spaying. The cost of breeding (as opposed to spaying) in period $t-1$ is $r+c_{l}-c_{s}$, while the benefit in period $t$ is $x q_{p}$. The price $q_{p}$ is such that $r+c_{l}-c_{s}=\delta x q_{p}$, which makes owners indifferent. Note that a high type has strictly less incentive to have a mixed breed litter, for, in the event that the parent pet dies, a high type owner that keeps his mixed breed puppy or kitten will forego the ownership of a young pure breed, which will create a loss of surplus. The postulated breeding and spaying behavior is therefore consistent with optimization, with the caveat that it is mixed breed owners who are generating the supply of young mixed breeds.

The second condition is that new owners will choose pets in a way consistent with $\left(\mathbf{H}_{t}^{n}, \mathbf{L}_{t}^{n}\right)$. Thus, we must show that high type new owners purchase young pure breeds and low types acquire young mixed breeds. We begin with high type new owners. Consider such an owner at the beginning of some period $t \geq 2$ and assume first that he does not own any young pets. Let $V_{H}(1)$ denote his expected equilibrium payoff. In addition, let $V_{H}(0)$ denote the equilibrium payoff of a high type owner at the beginning of some period $t \geq 2$ who does not and cannot own a pet that

\footnotetext{
34 To preserve space, many details are omitted from these "proofs". Full length proofs are available from the authors on request.
} 
period. Under the proposed equilibrium behavior, our owner purchases a young pure breed and thus

$$
\begin{gathered}
V_{H}(1)=\beta+\theta-q_{p}-c_{s}+\delta\left[\eta\left\{\alpha(\beta+\theta)-(1-\alpha) \xi_{o}+\delta\left(\alpha V_{H}(1)+(1-\alpha) V_{H}(0)\right)\right\}\right. \\
\left.+(1-\eta)\left(\alpha V_{H}(1)+(1-\alpha) V_{H}(0)\right)\right] .
\end{gathered}
$$

This expression reflects the facts that: i) a young pure breed costs $q_{p}$, ii) the owner is indifferent between spaying and breeding and thus can be assumed to spay, iii) a young pet survives to be old with probability $\eta$, iv) the owner will keep his old pet with probability $\alpha$, and, v) if the owner is unable to keep his pet it will be euthanized. Since a potential owner who does not and cannot own a pet gets no contemporaneous pet-related benefit, $V_{H}(0)=\delta\left(\alpha V_{H}(1)+(1-\alpha) V_{H}(0)\right)$, which implies that

$$
V_{H}(0)=\left(\frac{\delta \alpha}{1-\delta(1-\alpha)}\right) V_{H}(1)
$$

Substituting (28) in to (27), the equilibrium payoff of our owner is

$$
V_{H}(1)=\left[\beta+\theta-q_{p}-c_{s}+\delta \eta\left\{\alpha(\beta+\theta)-(1-\alpha) \xi_{o}\right\}\right] \frac{1-\delta(1-\alpha)}{(1-\delta)(1+\eta \alpha \delta)} .
$$

We must show that the owner cannot achieve a higher payoff by deviating from his proposed equilibrium behaviour. It suffices to consider one shot deviations and there are three such deviations to study. Suppose first that he decides not to own any pet. Then his payoff under the deviation is $\delta\left(\alpha V_{H}(1)+(1-\alpha) V_{H}(0)\right)$. Using (28), we have that

$$
V_{H}(1)-\delta\left(\alpha V_{H}(1)+(1-\alpha) V_{H}(0)\right)=V_{H}(1)\left(\frac{1-\delta}{1-\delta(1-\alpha)}\right),
$$

which is positive as long as $V_{H}(1)>0$. From (10) and (29), this is the case under Assumptions 2 and 3. Next suppose that our owner decides to obtain a young mixed breed from the shelter. This yields a payoff of

$$
\beta-c_{s}+\delta\left[\eta\left\{\alpha \beta-(1-\alpha) \xi_{o}+\delta\left(\alpha V_{H}(1)+(1-\alpha) V_{H}(0)\right)\right\}+(1-\eta)\left(\alpha V_{H}(1)+(1-\alpha) V_{H}(0)\right)\right] .
$$

Using (27), for the deviation to be unprofitable, we need that $\theta(1+\delta \eta \alpha) \geq q_{p}$. This follows from (10) and Assumption 3. Finally, suppose that he decides to pick up an old pure breed from the shelter. The payoff from this is $\lambda(\beta+\theta)+\delta\left(\alpha V_{H}(1)+(1-\alpha) V_{H}(0)\right)$. Using (28), we have that

$$
V_{H}(1)-\left[\lambda(\beta+\theta)+\delta\left(\alpha V_{H}(1)+(1-\alpha) V_{H}(0)\right)\right]=V_{H}(1)\left(\frac{1-\delta}{1-\delta(1-\alpha)}\right)-\lambda(\beta+\theta) .
$$


From (29), this difference is non-negative if

$$
\lambda(\beta+\theta) \leq\left[\beta+\theta-q_{p}-c_{s}+\delta \eta\left\{\alpha(\beta+\theta)-(1-\alpha) \xi_{o}\right\}\right] \frac{1}{(1+\eta \alpha \delta)} .
$$

Using (10), this is true under Assumption 4(ii).

If the owner owns young pets they will be pure breeds. His expected equilibrium payoff will be $V_{H}(1)+x q_{p}$. Since the payoffs from deviating from his proposed equilibrium behavior are also altered by simply adding $x q_{p}$, the earlier arguments apply unchanged.

Now consider low type new owners. Consider such an owner at the beginning of some period $t \geq 2$ and assume first that he does not own any young pets. Let $V_{L}(1)$ denote his expected equilibrium payoff. Similarly, let $V_{L}(0)$ denote the equilibrium payoff of a low type owner at the beginning of some period $t \geq 2$ who does not and cannot own a pet that period. Under the proposed equilibrium behavior, our owner obtains a young mixed breed from the shelter and thus

$$
\begin{aligned}
V_{L}(1)=\beta-c_{s}+ & \delta\left[\eta\left\{\alpha \beta-(1-\alpha) \xi_{o}+\delta\left(\alpha V_{L}(1)+(1-\alpha) V_{L}(0)\right)\right\}\right. \\
+ & \left.(1-\eta)\left(\alpha V_{L}(1)+(1-\alpha) V_{L}(0)\right)\right] .
\end{aligned}
$$

Again, this assumes that the owner spays his pet which is justified by the indifference between spaying and not. As shown for high types

$$
V_{L}(0)=\left(\frac{\delta \alpha}{1-\delta(1-\alpha)}\right) V_{L}(1)
$$

and therefore the equilibrium payoff of the owner is

$$
V_{L}(1)=\left[\beta-c_{s}+\delta \eta\left\{\alpha \beta-(1-\alpha) \xi_{o}\right\}\right] \frac{1-\delta(1-\alpha)}{(1-\delta)(1+\eta \alpha \delta)} .
$$

Again, we must show that the owner cannot achieve a higher payoff by deviating from his proposed equilibrium behaviour. Suppose first that he decides not to own any pet. Then his payoff under the deviation is $\delta\left(\alpha V_{L}(1)+(1-\alpha) V_{L}(0)\right)$. Using (31), we have that

$$
V_{L}(1)-\delta\left(\alpha V_{L}(1)+(1-\alpha) V_{L}(0)\right)=V_{L}(1)\left(\frac{1-\delta}{1-\delta(1-\alpha)}\right)
$$

which is positive as long as $V_{L}(1)>0$. From (32), this is the case under Assumption 2. Next suppose that our owner decides to purchase a young pure breed. This yields a payoff of

$\beta-q_{p}-c_{s}+\delta\left[\eta\left\{\alpha \beta-(1-\alpha) \xi_{o}+\delta\left(\alpha V_{L}(1)+(1-\alpha) V_{L}(0)\right)\right\}+(1-\eta)\left(\alpha V_{L}(1)+(1-\alpha) V_{L}(0)\right)\right]$. 
Using (30), for this deviation to be unprofitable, we need only that $q_{p}>0$. Finally, suppose that he decides to pick up an old pet from the shelter. The payoff from deviating is $\lambda \beta+\delta\left(\alpha V_{L}(1)+\right.$ $\left.(1-\alpha) V_{L}(0)\right)$. Using $(31)$

$$
V_{L}(1)-\lambda \beta-\delta\left(\alpha V_{L}(1)+(1-\alpha) V_{L}(0)\right)=V_{L}(1)\left(\frac{1-\delta}{1-\delta(1-\alpha)}\right)-\lambda \beta .
$$

From (32), this is non-negative if

$$
\lambda \beta \leq\left(\beta-c_{s}+\delta \eta\left\{\alpha \beta-(1-\alpha) \xi_{o}\right\}\right) \frac{1}{(1+\eta \alpha \delta)} .
$$

This is true under Assumption 4(i).

If the owner owns young pets then they must be mixed breeds. His expected equilibrium payoff will be $V_{L}(1)-(x-1) \pi_{m} \xi_{y}$. Since the payoffs from deviating from his proposed equilibrium behavior are altered by subtracting $x \pi_{m} \xi_{y}$, the earlier arguments remain valid.

The third condition is that supply equals demand for any pet type whose price is positive. The only pet type with a positive price is young pure breed. All new owners who are high types demand young pure breeds and hence demand in period $t \geq 2$ is $\gamma_{H}-\alpha H_{t}^{o}$. By construction, the supply is $y_{p t}=\gamma_{H}-\alpha H_{t}^{o}$ and so the condition is satisfied.

The fourth condition is that supply exceeds demand for any pet type with a zero price and the actual euthanization probabilites are equal to $\boldsymbol{\pi}_{t}$. The only thing to show is that the probability that a young mixed breed taken to the shelter is euthanized is given by (11). Let $t \geq 2$ be given. The number of low type owners of young pets in period $t-1$ is $L_{t}^{o} / \eta$. Let $\kappa_{t-1}$ denote the fraction of these owners who do not spay their pets in period $t-1$. Since all high type owners will either spay or breed their pets, the number of young mixed breeds in period $t$ is $\varphi x \kappa_{t-1} L_{t}^{o} / \eta$. Since it must be the case that $y_{m t}=\varphi x \kappa_{t-1} L_{t}^{o} / \eta$, it follows that $\kappa_{t-1}=y_{m t} /\left(\varphi x L_{t}^{o} / \eta\right)$. The number of young mixed breeds taken to the shelter in period $t$ is

$$
\varphi\{x-(1-\eta) \alpha\} \kappa_{t-1} L_{t}^{o} / \eta=\{x-(1-\eta) \alpha\} \frac{y_{m t}}{x} .
$$

The demand for shelter pets in period $t$ is

$$
\gamma_{L}-\alpha\left\{\eta+(1-\eta) \kappa_{t-1} \varphi\right\} L_{t}^{o} / \eta=\gamma_{L}-\alpha\left\{\eta+(1-\eta) \frac{y_{m t}}{\varphi x L_{t}^{o} / \eta} \varphi\right\} L_{t}^{o} / \eta
$$

Thus, the probability that a pet taken to the shelter is euthanized is

$$
\frac{\{x-(1-\eta) \alpha\} \frac{y_{m t}}{x}-\left[\gamma_{L}-\alpha\left\{\eta+(1-\eta) \frac{y_{m t}}{\varphi x L_{t}^{o} / \eta} \varphi\right\} L_{t}^{o} / \eta\right]}{\{x-(1-\eta) \alpha\} \frac{y_{m t}}{x}}=\frac{y_{m t}-\left[\gamma_{L}-\alpha L_{t}^{o}\right]}{\{x-(1-\eta) \alpha\} \frac{y_{m t}}{x}} .
$$


Recalling that $y_{m t}=\varphi x\left(\gamma_{L}-\alpha L_{t}^{o}\right) /\left[\varphi x-\left(c_{s}-\varphi c_{l}\right) / \delta \xi_{y}\right]$ yields the result.

It remains to show that given the dynamics of the equilibrium, the pet populations converge to the steady state (13). From (12), for all $t \geq 2,\left(y_{p t+1}, y_{m t+1}\right)=\left(\gamma_{H}-\alpha \eta y_{p t}, \frac{\gamma_{L}}{1-\left(c_{s}-\varphi c_{l}\right) / \delta \varphi x \xi_{y}}-\right.$ $\left.\alpha \eta y_{m t}\right)$. This is a linear system of difference equations which can be written in matrix form as

$$
\left[\begin{array}{c}
y_{p t+1} \\
y_{m t+1}
\end{array}\right]=\left[\begin{array}{cc}
-\alpha \eta & 0 \\
0 & -\alpha \eta
\end{array}\right]\left[\begin{array}{c}
y_{p t} \\
y_{m t}
\end{array}\right]+\left[\begin{array}{c}
\gamma_{H} \\
\frac{\gamma_{L}}{1-\left(c_{s}-\varphi c_{l}\right) / \delta \varphi x \xi_{y}}
\end{array}\right] .
$$

A sufficient condition for stability is that all the characteristic roots of the matrix have moduli strictly less than 1 (Sydsaeter (1981), Theorem 7.7). A sufficient condition for this is that the sum of the absolute value of the rows of the matrix be less than 1 (Sydsaeter (1981), Theorem 7.8). Since this is satisfied, we conclude that the system converges to its steady state, which is (13).

\subsection{Proof of Proposition 2}

Let $\left(\mathbf{H}^{n *}, \mathbf{L}^{n *}, \mathbf{y}^{*}\right)$ be the steady state of the efficient plan. We will make extensive use of the fact that $\mathbf{y}^{*}, \mathbf{H}_{y}^{n *}, \mathbf{L}_{y}^{n *}, \mathbf{H}_{o}^{n *}, \mathbf{L}_{o}^{n *}, \mathbf{H}_{o}^{n *}$ and $\mathbf{L}_{o}^{n *}$ must solve the problem of choosing $\mathbf{y}, \mathbf{H}_{y}^{n}, \mathbf{L}_{y}^{n}, \mathbf{H}_{o 1}^{n}$, $\mathbf{L}_{o 1}^{n}, \mathbf{H}_{o 2}^{n}$ and $\mathbf{L}_{o 2}^{n}$ to maximize the objective function

$$
\begin{gathered}
S\left(\eta \mathbf{H}_{y}^{n *}, \eta \mathbf{L}_{y}^{n *}, \mathbf{y}^{*}, \mathbf{H}^{n *}, \mathbf{L}^{n *}, \mathbf{y}\right)+\delta S\left(\eta \mathbf{H}_{y}^{n *}, \eta \mathbf{L}_{y}^{n *}, \mathbf{y},\left(\mathbf{H}_{y}^{n}, \mathbf{H}_{o 1}^{n}\right),\left(\mathbf{L}_{y}^{n}, \mathbf{L}_{o 1}^{n}\right), \mathbf{y}^{*}\right) \\
+\delta^{2} S\left(\eta \mathbf{H}_{y}^{n}, \eta \mathbf{L}_{y}^{n}, \mathbf{y}^{*},\left(\mathbf{H}_{y}^{n *}, \mathbf{H}_{o 2}^{n}\right),\left(\mathbf{L}_{y}^{n *}, \mathbf{L}_{o 2}^{n}\right), \mathbf{y}^{*}\right)
\end{gathered}
$$

subject to the feasibility constraints

$$
\begin{gathered}
H_{y p}^{n}+H_{y m}^{n}+H_{o p 1}^{n}+H_{o m 1}^{n} \leq \gamma_{H}-\alpha \eta\left(H_{y p}^{n *}+H_{y m}^{n *}\right) \\
L_{y p}^{n}+L_{y m}^{n}+L_{o p 1}^{n}+L_{o m 1}^{n} \leq \gamma_{L}-\alpha \eta\left(L_{y p}^{n *}+L_{y m}^{n *}\right) \\
H_{y p}^{n}+L_{y p}^{n} \leq y_{p} \\
H_{y m}^{n}+L_{y m}^{n} \leq y_{m} \\
H_{o p 1}^{n}+L_{o p 1}^{n} \leq(1-\alpha) \eta\left(H_{y p}^{n *}+L_{y p}^{n *}\right) \\
H_{o m 1}^{n}+L_{o m 1}^{n} \leq(1-\alpha) \eta\left(H_{y m}^{n *}+L_{y m}^{n *}\right),
\end{gathered}
$$


the feasibility constraints

$$
\begin{gathered}
H_{y p}^{n *}+H_{y m}^{n *}+H_{o p 2}^{n}+H_{o m 2}^{n} \leq \gamma_{H}-\alpha \eta\left(H_{y p}^{n}+H_{y m}^{n}\right) \\
L_{y p}^{n *}+L_{y m}^{n *}+L_{o p 2}^{n}+L_{o m 2}^{n} \leq \gamma_{L}-\alpha \eta\left(L_{y p}^{n}+L_{y m}^{n}\right) \\
H_{o p 2}^{n}+L_{o p 2}^{n} \leq(1-\alpha) \eta\left(H_{y p}^{n}+L_{y p}^{n}\right) \\
H_{o m 2}^{n}+L_{o m 2}^{n} \leq(1-\alpha) \eta\left(H_{y m}^{n}+L_{y m}^{n}\right),
\end{gathered}
$$

and the constraint that all choice variables must be non-negative.

To interpret this problem, think of $\mathbf{y}$ as the number of young pets in some period $t ; \mathbf{H}_{y}^{n}$ and $\mathbf{L}_{y}^{n}$ the numbers of young pets allocated to new owners in period $t ; \mathbf{H}_{o 1}^{n}$ and $\mathbf{L}_{o 1}^{n}$ the numbers of old relinquished pets allocated to new owners in period $t$; and $\mathbf{H}_{o 2}^{n}$ and $\mathbf{L}_{o 2}^{n}$ the numbers of old relinquished pets allocated to new owners in period $t+1$. The first set of feasibility constraints (34) reflect those arising in period $t$ and the second set (35) reflect those arising in period $t+1$. We will refer to this problem as the efficiency problem and to $\mathbf{y}^{*}, \mathbf{H}_{y}^{n *}, \mathbf{L}_{y}^{n *}, \mathbf{H}_{o}^{n *}, \mathbf{L}_{o}^{n *}, \mathbf{H}_{o}^{n *}$ and $\mathbf{L}_{o}^{n *}$ as the proposed solution.

Lemma 1 Suppose that Assumption 5 is satisfied and that the efficient plan converges to a steady state. Then, in this steady state, no young pets are euthanized.

Proof: We need to show that $H_{y i}^{n *}+L_{y i}^{n *}=y_{i}^{*}$ for $i \in\{p, m\}$. We only prove the result for mixed breeds, the argument for pure breeds being similar. Suppose that, $H_{y m}^{n *}+L_{y m}^{n *}<y_{m}^{*}$. Consider perturbing the proposed solution by decreasing $y_{m}$ to $y_{m}^{*}-\Delta$ where $\Delta$ is small and positive. This change clearly satisfies the feasibility constraints (34) and (35) for $\Delta$ sufficiently small. Denote the value of the objective function (33) as a function of $\Delta$ as $S^{*}(\Delta)$. Using (4)-(8), we find that

$$
\frac{d S^{*}(0)}{d \Delta}=-\left(\frac{c_{l}}{x}-\frac{c_{s}}{\varphi x}\right)+\delta\left(\xi_{y}+\rho\right) .
$$

This change is positive under Assumption 5 - which contradicts the fact that the proposed solution must solve the efficiency problem.

Lemma 2 Suppose that Assumption 2 is satisfied and that the efficient plan converges to a steady state. Then, in this steady state, all new owners have pets.

Proof: We need to show that $L_{y p}^{n *}+L_{y m}^{n *}+L_{o p}^{n *}+L_{o m}^{n *}=\gamma_{L}-\alpha \eta\left(L_{y p}^{n *}+L_{y m}^{n *}\right)$ and that $H_{y p}^{n *}+$ $H_{y m}^{n *}+H_{o p}^{n *}+H_{o m}^{n *}=\gamma_{H}-\alpha \eta\left(H_{y p}^{n *}+H_{y m}^{n *}\right)$. We show only the first equality, the argument for the second being similar. Suppose that $L_{y p}^{n *}+L_{y m}^{n *}+L_{o p}^{n *}+L_{o m}^{n *}<\gamma_{L}-\alpha \eta\left(L_{y p}^{n *}+L_{y m}^{n *}\right)$. Consider the 
following perturbation of the proposed solution: increase $y_{m}$ to $y_{m}^{*}+\Delta, L_{y m}^{n}$ to $L_{y m}^{n *}+\Delta$, and $L_{o m}^{n}$ to $L_{o m}^{n *}+(1-\alpha) \eta \Delta$ where $\Delta$ is small and positive. This perturbation satisfies the feasibility constraints (34) and (35) for $\Delta$ sufficiently small. Denote the value of the objective function (33) as a function of $\Delta$ as $S^{*}(\Delta)$. Using (4)-(8), we find that

$$
\frac{d S^{*}(0)}{d \Delta}=-\left(\frac{c_{l}}{x}-\frac{c_{s}}{\varphi x}\right)+\delta \beta+\delta^{2} \eta(\alpha \beta+(1-\alpha) \lambda \beta) .
$$

This is positive under Assumption 2, which contradicts the fact that the proposed solution solves the efficiency problem.

Lemma 3 Suppose that Assumptions 2 and 3 are satisfied and that the efficient plan converges to a steady state. Then, in this steady state, any new owner who receives a young pet has a pure breed if he is a high type and a mixed breed if he is a low type.

Proof: By Lemma 1, we know that $H_{y p}^{n *}+L_{y p}^{n *}=y_{p}^{*}$ and that $H_{y m}^{n *}+L_{y m}^{n *}=y_{m}^{*}$. We need to show that $L_{y p}^{n *}=0$ and $H_{y m}^{n *}=0$ which would imply that $\left(H_{y p}^{n *}, H_{y m}^{n *}, L_{y p}^{n *}, L_{y m}^{n *}\right)=\left(y_{p}^{*}, 0,0, y_{m}^{*}\right)$. We show only the first equality, the argument for the second being similar. Suppose that $L_{y p}^{n *}>0$. Consider the following perturbation of the proposed solution: reduce $y_{p}$ to $y_{p}^{*}-\Delta$, increase $y_{m}$ to $y_{m}^{*}+\Delta$, decrease $L_{y p}^{n}$ to $L_{y p}^{n *}-\Delta$, increase $L_{y m}^{n}$ to $L_{y m}^{n *}+\Delta$, decrease $L_{o p}^{n}$ to $L_{o p}^{n *}-(1-\alpha) \eta \Delta$, and increase $L_{o m}^{n}$ to $L_{o m}^{n *}+(1-\alpha) \eta \Delta$ where $\Delta$ is small and positive. This change is feasible for sufficiently small $\Delta$ provided that $L_{o p}^{n *}>0$, which we assume for now. Denote the value of the objective function (33) as a function of $\Delta$ as $S^{*}(\Delta)$. Using (4)-(8), we find that

$$
\frac{d S^{*}(0)}{d \Delta}=\frac{r}{x}+\frac{c_{s}(1-\varphi)}{\varphi x}
$$

This is positive, which contradicts the fact that the proposed solution solves the efficiency problem.

The above analysis assumes that $L_{o p}^{n *}>0$. If $L_{o p}^{n *}=0$, there are two possibilities. The first is that $H_{o p}^{n *}<(1-\alpha) \eta\left(H_{y p}^{n *}+L_{y p}^{n *}\right)$, so that some old relinquished pure breeds are euthanized. In this case, in the above perturbation, we set $L_{o p}^{n}=L_{o p}^{n *}$ and $L_{o m}^{n}=L_{o m}^{n *}$. This change is feasible, since all that happens is that old relinquished mixed breeds are euthanized instead of pure breeds, and this has no implications for payoffs. The second possibility is that $H_{o p}^{n *}=(1-\alpha) \eta\left(H_{y p}^{n *}+L_{y p}^{n *}\right)$. However, with a little work, this can be shown to be impossible.

Lemma 4 Suppose that Assumptions 2, 3, 5, and 6 are satisfied and that the efficient plan converges to a steady state. Then, in this steady state, relinquished old pets are not euthanized. 
Proof: We need to show that $H_{o m}^{n *}+L_{o m}^{n *}=(1-\alpha) \eta\left(H_{y m}^{n *}+L_{y m}^{n *}\right)$ and $H_{o p}^{n *}+L_{o p}^{n *}=(1-\alpha) \eta\left(H_{y p}^{n *}+\right.$ $\left.L_{y p}^{n *}\right)$. We show only the first equality, the argument for the second being similar. Suppose that $H_{o m}^{n *}+L_{o m}^{n *}<(1-\alpha) \eta\left(H_{y m}^{n *}+L_{y m}^{n *}\right)$. Consider the following perturbation of the proposed solution: decrease $y_{m}$ to $y_{m}^{*}-\Delta$, decrease $L_{y m}^{n}$ to $L_{y m}^{n *}-\Delta$, increase $L_{o m 1}^{n}$ to $L_{o m}^{n *}+\Delta$, and increase $L_{o m 2}^{n}$ to $L_{o m}^{n *}+\alpha \eta \Delta$ where $\Delta$ is small and positive. This perturbation satisfies the feasibility constraints (34) and (35) for $\Delta$ sufficiently small. Denote the value of the objective function (33) as a function of $\Delta$ as $S^{*}(\Delta)$. Using (4)-(8), we find that

$$
\frac{d S^{*}(0)}{d \Delta}=\left(\frac{c_{l}}{x}-\frac{c_{s}}{\varphi x}\right)+\delta\left[c_{s}+\xi_{o}+\rho-\beta(1-\lambda)\right]+\eta \delta^{2}\left[\xi_{o}+\rho-\alpha \beta(1-\lambda)\right] .
$$

This is positive under Assumption 6(i) - which contradicts the fact that the proposed solution solves the efficiency problem.

Lemma 5 Suppose that Assumptions 2, 3, 5, and 6 are satisfied and that the efficient plan converges to a steady state. Then, in the efficient steady state, any new owner who receives a relinquished old pet has a pure breed if he is a high type.

Proof: We need to show that $H_{o m}^{n *}=0$. Suppose that $H_{o m}^{n *}>0$. Consider the following perturbation of the proposed solution: increase $y_{p}$ to $y_{p}^{*}+\Delta$, reduce $y_{m}$ to $y_{m}^{*}-\Delta$, increase $H_{y p}^{n}$ to $H_{y p}^{n *}+\Delta$, reduce $L_{y m}^{n}$ to $L_{y m}^{n *}-\Delta$, reduce $H_{o m 1}^{n}$ to $H_{o m}^{n *}-\Delta$, increase $L_{o m 1}^{n}$ to $L_{o m}^{n *}+\Delta$, increase $H_{o p 2}^{n}$ to $H_{o p 2}^{n *}+(1-\alpha) \eta \Delta$, decrease $H_{o m 2}^{n}$ to $H_{o m 2}^{n *}-\eta \Delta$, and increase $L_{o m 2}^{n}$ to $L_{o m 2}^{n *}+\alpha \eta \Delta$ where $\Delta$ is small and positive. This perturbation satisfies the feasibility constraints (34) and (35) for $\Delta$ sufficiently small. Denote the value of the objective function (33) as a function of $\Delta$ as $S^{*}(\Delta)$. Using (4)-(8), we find that

$$
\frac{d S^{*}(0)}{d \Delta}=-\left[\frac{r}{x}+\frac{c_{s}}{x}\left(\frac{1-\varphi}{\varphi}\right)\right]+\delta \theta+\delta^{2}[\alpha \eta \theta+(1-\alpha) \eta \lambda \theta] .
$$

This is positive by Assumption 3 - which contradicts the fact that the proposed solution solves the efficiency problem.

Summarizing the results so far, we have that $\left(H_{y p}^{n *}, H_{y m}^{n *}, L_{y p}^{n *}, L_{y m}^{n *}\right)=\left(y_{p}^{*}, 0,0, y_{m}^{*}\right)$ and that $H_{o m}^{n *}=0$. We also know that $H_{o p}^{n *}+L_{o p}^{n *}=(1-\alpha) \eta y_{p}^{*}$ and $L_{o m}^{n *}=(1-\alpha) \eta y_{m}^{*}$. Moreover, we know that $(1+\alpha \eta) y_{p}^{*}+H_{o p}^{n *}=\gamma_{H}$ and $(1+\alpha \eta) y_{m}^{*}+L_{o p}^{n *}+L_{o m}^{n *}=\gamma_{L}$. The only remaining issue is what is $H_{o p}^{n *}$ ? All we know is that $H_{o p}^{n *} \in\left[0,(1-\alpha) \eta y_{p}^{*}\right]$. Once we have pinned down $H_{o p}^{n *}$ more precisely we will have a complete picture of the steady state values of all the endogenous variables. 
To determine the value of $H_{o p}^{n *}$ we follow the usual procedure. Suppose that $H_{o p}^{n *}>0$. Consider the following perturbation of the proposed solution: increase $y_{p}$ to $y_{p}^{*}+\Delta$, decrease $y_{m}$ to $y_{m}^{*}-\Delta$, increase $H_{y p}^{n}$ to $H_{y p}^{n *}+\Delta$, decrease $L_{y m}^{n}$ to $L_{y m}^{n *}-\Delta$, decrease $H_{o p 1}^{n}$ to $H_{o p}^{n *}-\Delta$, increase $L_{o p 1}^{n}$ to $L_{o p}^{n *}+\Delta$, decrease $H_{o p 2}^{n}$ to $H_{o p 2}^{n *}-\alpha \eta \Delta$, increase $L_{o p 2}^{n}$ to $L_{o p 2}^{n *}+\eta \Delta$, and decrease $L_{o m 2}^{n}$ to $L_{o m 2}^{n *}-$ $(1-\alpha) \eta \Delta$ where $\Delta$ is small and positive. This perturbation satisfies the feasibility constraints (34) and (35) for $\Delta$ sufficiently small. Denote the value of the objective function (33) as a function of $\Delta$ as $S^{*}(\Delta)$. Using (4)-(8), we find that

$$
\frac{d S^{*}(0)}{d \Delta}=-\left[\frac{r}{x}+\frac{c_{s}}{x}\left(\frac{1-\varphi}{\varphi}\right)\right]+\delta \theta(1-\lambda)+\delta^{2} \alpha \eta \theta(1-\lambda) .
$$

The sign of this derivative is negative if (14) is satisfied and positive if it is not. The proposed perturbation involves a reduction in $H_{o p}^{n *}$. If this derivative is negative, then it must be the case that increasing $H_{o p}^{n *}$ by reversing this perturbation raises surplus. In that case, therefore, $H_{o p}^{n *}=(1-\alpha) \eta y_{p}^{*}$. On the other hand, if the derivative is positive, then $H_{o p}^{n *}=0$.

Suppose therefore that (14) is satisfied so that $H_{o p}^{n *}=(1-\alpha) \eta y_{p}^{*}$. Then given our previous results, it is easy to show that $\left(y_{p}^{*}, y_{m}^{*}\right)=\left(\frac{\gamma_{H}}{1+\eta}, \frac{\gamma_{L}}{1+\eta}\right)$, which is the first part of Proposition 2. On the other hand, if (14) is not satisfied, so that $H_{o p}^{n *}=0$ then $\left(y_{p}^{*}, y_{m}^{*}\right)=\left(\frac{\gamma_{H}}{1+\alpha \eta}, \frac{\gamma_{L}(1+\alpha \eta)-(1-\alpha) \eta \gamma_{H}}{(1+\eta)(1+\alpha \eta)}\right)$, which is the second part of Proposition 2.

\subsection{Proof of Proposition 3}

There are three things to establish. First, that the allocation and production plan described in the text is a market equilibrium under the subsidy (18) and the tax (19). Second, that given the dynamics of the equilibrium, the pet populations converge to the steady state (15). Third, that lifetime surplus in this equilibrium exceeds that generated by the unregulated equilibrium described in Proposition 1.

\subsubsection{The plan is an equilibrium}

Proving this follows the same basic steps as the proof of Proposition 1 and thus we will be brief. To see that the proposed allocation and production plan satisfies the first condition for equilibrium, note that the price $q_{y p}$ is such that $r+c_{l}-\left(c_{s}-s_{s}\right)=\delta x q_{y p}$, which makes owners of pure breeds indifferent between breeding or spaying. In addition, given that the price of young mixed breeds is 0 and the post-subsidy price of spaying is $c_{s}-s_{s}=\varphi c_{l}$, owners of all young pets will be indifferent between spaying or not. The third and fourth conditions are also easily verified. 
It remains to verify the second condition that new owners will choose pets in a way consistent with the proposed equilibrium. We begin with high type new owners. Consider such an owner at the beginning of some period $t \geq 2$ and assume first that he does not own any young pets. Let $V_{H}(1)$ denote his expected equilibrium payoff. Similarly, let $V_{H}(0)$ denote the equilibrium payoff of a high type owner at the beginning of some period $t \geq 2$ who does not and cannot own a pet that period. Under the proposed equilibrium behavior, the owner acquires a young pure breed and thus

$$
\begin{gathered}
V_{H}(1)=\beta+\theta-q_{y p}-\left(c_{s}-s_{s}\right)-T_{y}+\delta\left[\eta\left\{\alpha(\beta+\theta)+\delta\left(\alpha V_{H}(1)+(1-\alpha) V_{H}(0)\right)\right\}\right. \\
\left.+(1-\eta)\left(\alpha V_{H}(1)+(1-\alpha) V_{H}(0)\right)\right] .
\end{gathered}
$$

Since

$$
V_{H}(0)=\left(\frac{\delta \alpha}{1-\delta(1-\alpha)}\right) V_{H}(1)
$$

the equilibrium payoff of the owner is

$$
V_{H}(1)=\left[\beta+\theta-q_{y p}-\left(c_{s}-s_{s}\right)-T_{y}+\delta \eta \alpha(\beta+\theta)\right] \frac{1-\delta(1-\alpha)}{(1-\delta)(1+\eta \alpha \delta)} .
$$

Using this expression, (18), (19), (20) and the fact that (14) is satisfied, it is now straightforward to show that the owner cannot achieve a higher payoff by deviating from his proposed equilibrium behaviour. If he owns young pets, then his equilibrium payoff will be unchanged if they are mixed breeds and increased by $x q_{y p}$ if they are pure breeds. Since the same is true for the payoffs from deviating, the arguments remain valid in this case.

Now consider low type new owners. Consider such an owner at the beginning of some period $t \geq 2$ and assume first that he does not own any young pets. Let $V_{L}(1)$ denote his expected equilibrium payoff. Similarly, let $V_{L}(0)$ denote the equilibrium payoff of a low type owner at the beginning of some period $t \geq 2$ who does not and cannot own a pet that period. Under the prescribed equilibrium behavior, the owner either obtains a young mixed breeds or an old pet from the shelter. In particular, then

$$
\begin{gathered}
V_{L}(1)=\beta-\left(c_{s}-s_{s}\right)-T_{y}+\delta\left[\eta\left\{\alpha \beta+\delta\left(\alpha V_{L}(1)+(1-\alpha) V_{L}(0)\right)\right\}\right. \\
\left.+(1-\eta)\left(\alpha V_{L}(1)+(1-\alpha) V_{L}(0)\right)\right] .
\end{gathered}
$$


Since

$$
V_{L}(0)=\left(\frac{\delta \alpha}{1-\delta(1-\alpha)}\right) V_{L}(1)
$$

the equilibrium payoff of a low type new owner is

$$
V_{L}(1)=\left[\beta-\left(c_{s}-s_{s}\right)-T_{y}+\delta \eta \alpha \beta\right] \frac{1-\delta(1-\alpha)}{(1-\delta)(1+\eta \alpha \delta)} .
$$

Using this expression, (18), (19), and (20), it is now straightforward to show that the owner is indifferent between obtaining a young mixed breed or an old pet from the shelter, and that he cannot achieve a higher payoff by deviating from his proposed equilibrium behaviour. If he owns young pets, then they must be mixed breeds and his equilibrium payoffs will be unchanged. Since the same is true for the payoffs from deviating, the arguments remain valid in this case.

\subsubsection{Dynamics}

We know from (21) that for all $t \geq 2,\left(y_{p t+1}, y_{m t+1}\right)=\left(\gamma_{H}-\alpha \eta y_{p t}, \gamma_{L}-\eta y_{m t}-(1-\alpha) \eta y_{p t}\right)$. This is a linear system of difference equations that can be written in matrix form as

$$
\left[\begin{array}{c}
y_{p t+1} \\
y_{m t+1}
\end{array}\right]=\left[\begin{array}{cc}
-\alpha \eta & 0 \\
-(1-\alpha) \eta & -\eta
\end{array}\right]\left[\begin{array}{l}
y_{p t} \\
y_{m t}
\end{array}\right]+\left[\begin{array}{c}
\gamma_{H} \\
\gamma_{L}
\end{array}\right] .
$$

A sufficient condition for stability is that all the characteristic roots of the matrix have moduli strictly less than 1 . The characteristic roots of the matrix are the same as those of its transpose (Sydsaeter (1981)). The characteristic roots of the transposed matrix have moduli strictly less than 1 because the sum of the absolute value of its rows are less than 1 . Thus, the system converges to its steady state, which is (15).

\subsubsection{Surplus comparison}

Let $\left(\mathbf{H}_{t}^{n *}, \mathbf{L}_{t}^{n *}, \mathbf{y}_{t+1}^{*}\right)_{t=1}^{\infty}$ denote the equilibrium allocation and production plan described in Proposition 1 and let $\left(\mathbf{H}_{t}^{n e}, \mathbf{L}_{t}^{n e}, \mathbf{y}_{t+1}^{e}\right)_{t=1}^{\infty}$ denote the equilibrium allocation and production plan under the subsidy (18) and tax (19). To compute lifetime surplus under these plans, we need to specify what happens in the first period; that is, $\left(\mathbf{H}_{1}^{n *}, \mathbf{L}_{1}^{n *}\right)$ and $\left(\mathbf{H}_{1}^{n e}, \mathbf{L}_{1}^{n e}\right)$. By Assumption 7, the period 1 populations of young and old pets are such that $H_{m 1}^{o}=L_{p 1}^{o}=0, y_{p 1}=\gamma_{H}-\alpha H_{1}^{o}$, and $y_{m 1} \geq \gamma_{L}-\alpha L_{1}^{o}$. In this case, in either equilibrium, in period 1 the market will allocate all young pure breeds to high type new owners and low type new owners will get young mixed breeds. Thus, $\mathbf{H}_{1}^{n *}=\mathbf{H}_{1}^{n e}=\left(\gamma_{H}-\alpha H_{1}^{o}, 0,0,0\right)$ and $\mathbf{L}_{1}^{n *}=\mathbf{L}_{1}^{n e}=\left(0, \gamma_{L}-\alpha L_{1}^{o}, 0,0\right)$. 
Surplus in the market equilibrium Consider the lifetime payoffs of the different types of owners in the market equilibrium. There are $\gamma_{H}-\alpha H_{1}^{o}$ high type new owners in period 1. Assuming that they do not own any young pets at the beginning of period 1 , these owners obtain a lifetime surplus of

$\beta+\theta-q_{y p 1}^{*}-c_{s}+\delta\left[\eta\left\{\alpha(\beta+\theta)-(1-\alpha) \xi_{o}+\delta\left(\alpha V_{H}^{*}(1)+(1-\alpha) V_{H}^{*}(0)\right)\right\}+(1-\eta)\left(\alpha V_{H}^{*}(1)+(1-\alpha) V_{H}^{*}(0)\right)\right]$, where $V_{H}^{*}(1)$ and $V_{H}^{*}(0)$ are the equilibrium values defined in (28) and (29), and $q_{y p 1}^{*}$ is the equilibrium price of young pure breeds in period 1. This assumes the owner spays his pet in period 1, but, since the expected net benefits of either breeding or not spaying exactly equal $c_{s}$, the payoffs of new owners who either breed or do not spay their pets will be the same. There are also $\gamma_{L}-\alpha L_{1}^{o}$ low type new owners in period 1. Again, assuming they do not own any young pets at the beginning of period 1 , these owners obtain

$\beta-q_{y m 1}^{*}-c_{s}+\delta\left[\eta\left\{\alpha \beta-(1-\alpha) \xi_{o}+\delta\left(\alpha V_{L}^{*}(1)+(1-\alpha) V_{L}^{*}(0)\right)\right\}+(1-\eta)\left(\alpha V_{L}^{*}(1)+(1-\alpha) V_{L}^{*}(0)\right)\right]$.

where $V_{L}^{*}(1)$ and $V_{L}^{*}(0)$ are the equilibrium values defined in (31) and (32), and $q_{y m 1}^{*}$ is the equilibrium price of young mixed breeds in period 1 (which may be zero). Turning to old owners, there are $H_{1}^{o}$ high type old owners in period 1 who, assuming they do not own young pets, obtain a lifetime surplus of $\alpha(\beta+\theta)-(1-\alpha) \xi_{o}+\delta\left[\alpha V_{H}^{*}(1)+(1-\alpha) V_{H}^{*}(0)\right]$ and also $L_{1}^{o}$ low type old owners who obtain $\alpha \beta-(1-\alpha) \xi_{o}+\delta\left[\alpha V_{L}^{*}(1)+(1-\alpha) V_{L}^{*}(0)\right]$. Finally, there are owners who cannot own a pet in period 1 and do not own an old pet. There are $(1-\alpha)\left[\frac{\gamma_{H}}{\alpha}-H_{1}^{o}\right]$ high types in this group who, assuming that they do not own young pets at the beginning of period 1 , obtain a lifetime surplus of $\delta\left[\alpha V_{H}^{*}(1)+(1-\alpha) V_{H}^{*}(0)\right]$ and $(1-\alpha)\left[\frac{\gamma_{L}}{\alpha}-L_{1}^{o}\right]$ low types who obtain $\delta\left[\alpha V_{L}^{*}(1)+(1-\alpha) V_{L}^{*}(0)\right]$.

All the above expressions assume that owners do not own young pets at the beginning of period 1. If an owner does own young pets, we need to add on payments received for these pets and deduct psychic costs stemming from their euthanization. Notice that any payments are transfers and will offset the costs of purchasing young pets bourne by new owners. Thus, in an aggregate surplus calculation, all we need do is remove the period 1 prices from the above expressions for the new owners' surplus. The aggregate psychic costs from euthanizing young mixed breeds in period 1, must equal $\left[y_{m 1}-\left(\gamma_{L}-\alpha L_{1}^{o}\right)\right] \xi_{y}$ and so to account for these, we deduct this amount from the sum of new owners' payoffs. It is also necessary to account for the taxes necessary to 
finance the shelter. The discounted present value of these is

$\rho\left\{y_{m 1}-\left(\gamma_{L}-\alpha L_{1}^{o}\right)+\sum_{t=2}^{\infty} \delta^{t-1}\left(y_{m t}^{*}-\left(\gamma_{L}-\alpha L_{t}^{o *}\right)\right)+(1-\alpha)\left(L_{1}^{o}+H_{1}^{o}\right)+(1-\alpha) \sum_{t=2}^{\infty} \delta^{t-1}\left(L_{t}^{o *}+H_{t}^{o *}\right)\right\}$.

Aggregate lifetime surplus in the market equilibrium, denoted $S^{*}$, is now obtained by adding the lifetime payoffs of all the different types of owners described above (ignoring the first period prices) and subtracting the psychic costs of euthanizing surplus young mixed breeds in period 1 and the taxes necessary to finance the shelter.

Surplus in the corrected equilibrium Consider the payoffs of the different types of owners in the corrected equilibrium. There are $\gamma_{H}-\alpha H_{1}^{o}$ high type new owners in period 1. Assuming that they do not own any young pets at the beginning of period 1 , these owners obtain a lifetime surplus of

$\beta+\theta-q_{y p 1}^{e}-\varphi c_{l}+\delta\left[\eta\left\{\alpha(\beta+\theta)+\delta\left(\alpha V_{H}^{e}(1)+(1-\alpha) V_{H}^{e}(0)\right)\right\}+(1-\eta)\left(\alpha V_{H}^{e}(1)+(1-\alpha) V_{H}^{e}(0)\right)\right]$,

where $V_{H}^{e}(1)$ and $V_{H}^{e}(0)$ are defined in (37) and (38), and $q_{y p 1}^{e}$ is the equilibrium price of young pure breeds in period 1 . There are also $\gamma_{L}-\alpha L_{1}^{o}$ low type new owners in period 1 who obtain

$$
\beta-q_{y m 1}^{e}-\varphi c_{l}+\delta\left(\eta\left\{\alpha \beta+\delta\left[\alpha V_{L}^{e}(1)+(1-\alpha) V_{L}^{e}(0)\right)\right\}+(1-\eta)\left(\alpha V_{L}^{e}(1)+(1-\alpha) V_{L}^{e}(0)\right)\right]
$$

where $V_{L}^{e}(1)$ and $V_{L}^{e}(0)$ are defined in (40) and (41), and $q_{y m 1}^{e}$ is the equilibrium price of young mixed breeds in period 1. Turning to old owners, there are $H_{1}^{o}$ high type old owners in period 1 who, assuming that they do not own young pets, will obtain a lifetime surplus of $\alpha(\beta+\theta)-$ $(1-\alpha) \xi_{o}+\delta\left[\alpha V_{H}^{e}(1)+(1-\alpha) V_{H}^{e}(0)\right]$ and $L_{1}^{o}$ low type old owners who obtain $\alpha \beta-(1-\alpha) \xi_{o}+$ $\delta\left[\alpha V_{L}^{e}(1)+(1-\alpha) V_{L}^{e}(0)\right]$. Finally, there are potential owners who do not and cannot own a pet in period 1. There are $(1-\alpha)\left[\frac{\gamma_{H}}{\alpha}-H_{1}^{o}\right]$ high types in this group who, assuming that they do not own young pets, obtain a lifetime surplus of $\delta\left[\alpha V_{H}^{e}(1)+(1-\alpha) V_{H}^{e}(0)\right]$ and $(1-\alpha)\left[\frac{\gamma_{L}}{\alpha}-L_{1}^{o}\right]$ low types who obtain $\delta\left[\alpha V_{L}^{e}(1)+(1-\alpha) V_{L}^{e}(0)\right]$.

To obtain aggregate surplus, denoted $S^{e}$, we add up the lifetime payoffs of the different types of owners. Again, we deduct period 1 prices from the new owners' surplus since these are just transfers. We also subtract the psychic costs of euthanizing surplus young mixed breeds in period 1 (given by $\left.\left[y_{m 1}-\left(\gamma_{L}-\alpha L_{1}^{o}\right)\right] \xi_{y}\right)$ and the taxes necessary to finance the shelter. Since pets are euthanized only in period 1 , the latter are given by $\rho\left[y_{m 1}-\left(\gamma_{L}-\alpha L_{1}^{o}\right)+(1-\alpha)\left(L_{1}^{o}+H_{1}^{o}\right)\right]$. We 
also need to add the tax revenues that come from the tax on young pets. The present value of these is

$$
\sum_{t=2}^{\infty} \delta^{t-1} T_{y}\left(y_{m t}^{e}+y_{p t}^{e}\right)
$$

Finally, we need to deduct the discounted present value of the subsidy payments which is given by

$$
\left[1-\alpha\left(H_{1}^{o}+L_{1}^{o}\right)-\frac{y_{m 2}^{e}}{\varphi x}-\frac{y_{p 2}^{e}}{x}\right] s_{s}+\sum_{t=2}^{\infty} \delta^{t-1}\left[y_{p t}^{e}+y_{m t}^{e}-\frac{y_{m t+1}^{e}}{\varphi x}-\frac{y_{p t+1}^{e}}{x}\right] s_{s} .
$$

Comparing surplus The difference in surplus in the two equilibria may be written as

$$
S^{*}-S^{e}=S_{T}^{*}-S_{T}^{e}+S_{s}^{*}-S_{s}^{e},
$$

where $S_{T}^{*}-S_{T}^{e}$ is the difference in surplus resulting from the tax on young pets and $S_{s}^{*}-S_{s}^{e}$ is the difference resulting from the spaying subsidy. With a considerable amount of work, it can be shown that

$$
S_{T}^{*}-S_{T}^{e}=\frac{[\Delta(1-\delta)+\delta] \delta \eta(1-\alpha)}{(1-\delta)(1+\eta \alpha \delta)}\left\{\frac{T_{y}}{(1+\delta \eta)}-\left(\xi_{o}+\rho\right)\right\},
$$

where $\Delta=1-\alpha\left(H_{1}^{o}+L_{1}^{o}\right)$. Using (18), (19) and Assumption 6(i), we have that $T_{y} /(1+\delta \eta)-\left(\xi_{o}+\rho\right)$ is less than $s_{s} /(1+\delta \eta)$, so that

$$
S_{T}^{*}-S_{T}^{e}<\frac{[\Delta(1-\delta)+\delta] \delta \eta(1-\alpha)}{(1-\delta)(1+\eta \alpha \delta)(1+\delta \eta)} s_{s}
$$

Again with a considerable amount of work, we can also show that

$$
S_{s}^{*}-S_{s}^{e}<-\frac{[\Delta(1-\delta)+\delta] \delta \eta(1-\alpha)}{(1-\delta)(1+\eta \alpha \delta)(1+\delta \eta)} s_{s} .
$$

Combining (42) and (43), we have that

$$
S_{T}^{*}-S_{T}^{e}+S_{s}^{*}-S_{s}^{e}<\frac{[\Delta(1-\delta)+\delta] \delta \eta(1-\alpha)}{(1-\delta)(1+\eta \alpha \delta)(1+\delta \eta)} s_{s}-\frac{[\Delta(1-\delta)+\delta] \delta \eta(1-\alpha)}{(1-\delta)(1+\eta \alpha \delta)(1+\delta \eta)} s_{s}=0
$$

as required.

\subsection{Proof of Proposition 4}

There are three things to establish. First, that the allocation and production plan described in the text is a market equilibrium under the subsidy (18) and the taxes (22) and (23). Second, that given the dynamics of the equilibrium, the pet populations converge to the steady state (16). Third, that lifetime surplus in this equilibrium exceeds that generated by the laissez-faire equilibrium described in Proposition 1. 


\subsubsection{The plan is an equilibrium}

The argument that the proposed allocation and production plan satisfies the first condition for equilibrium is as in Proposition 3. The third and fourth conditions are also easily verified. It remains to show that new owners will choose pets in a way consistent with the proposed equilibrium. We begin with high type new owners. Consider such an owner at the beginning of some period $t \geq 2$ and assume first that he does not own any young pets. Let $V_{H}(1)$ denote his expected equilibrium payoff and let $V_{H}(0)$ denote the equilibrium payoff of a high type owner who does not and cannot own a pet. Under the proposed equilibrium behavior, the owner either acquires a young pure breed or gets an old pure breed from the shelter. In particular, then

$$
\begin{gathered}
V_{H}(1)=\beta+\theta-q_{y p}-\left(c_{s}-s_{s}\right)-T_{y p}+\delta\left[\eta\left\{\alpha(\beta+\theta)+\delta\left(\alpha V_{H}(1)+(1-\alpha) V_{H}(0)\right)\right\}\right. \\
\left.+(1-\eta)\left(\alpha V_{H}(1)+(1-\alpha) V_{H}(0)\right)\right]
\end{gathered}
$$

Since

$$
V_{H}(0)=\left(\frac{\delta \alpha}{1-\delta(1-\alpha)}\right) V_{H}(1)
$$

the equilibrium payoff of the owner is

$$
V_{H}(1)=\left[\beta+\theta-q_{y p}-\left(c_{s}-s_{s}\right)-T_{y p}+\delta \eta \alpha(\beta+\theta)\right] \frac{1-\delta(1-\alpha)}{(1-\delta)(1+\eta \alpha \delta)} .
$$

Using this expression, (20), (22), and (23), it is now straightforward to show that the owner is indifferent between purchasing a young pure breed and getting an old pure breed from the shelter, and cannot achieve a higher payoff by deviating from his proposed equilibrium behavior. If he owns young pets, then his equilibrium payoff will be unchanged if they are mixed breeds and increased by $x q_{y p}$ if they are pure breeds. Since the same is true for the payoffs from deviating, the arguments remain valid in this case.

Now consider low type new owners. Consider such an owner at the beginning of some period $t \geq 2$ and assume first that he does not own any young pets. Let $V_{L}(1)$ denote his expected equilibrium payoff and $V_{L}(0)$ the equilibrium payoff of a low type owner who does not and cannot own a pet. Under the prescribed equilibrium behavior, the owner either obtains a young mixed breed or an old pet from the shelter. In particular, then

$$
\begin{gathered}
V_{L}(1)=\beta-\left(c_{s}-s_{s}\right)-T_{y m}+\delta\left[\eta\left\{\alpha \beta+\delta\left(\alpha V_{L}(1)+(1-\alpha) V_{L}(0)\right)\right\}\right. \\
\left.+(1-\eta)\left(\alpha V_{L}(1)+(1-\alpha) V_{L}(0)\right)\right] .
\end{gathered}
$$


Since

$$
V_{L}(0)=\left(\frac{\delta \alpha}{1-\delta(1-\alpha)}\right) V_{L}(1)
$$

the equilibrium payoff of the owner is

$$
V_{L}(1)=\left[\beta-\left(c_{s}-s_{s}\right)-T_{y m}+\delta \eta \alpha \beta\right] \frac{1-\delta(1-\alpha)}{(1-\delta)(1+\eta \alpha \delta)} .
$$

Using this expression, (20), (22), and (23), it is now straightforward to show that the owner is indifferent between obtaining a young or old mixed breed and cannot achieve a higher payoff by deviating from his proposed equilibrium behavior. If he owns young pets, then they must be mixed breeds and his equilibrium payoffs will be unchanged. Since the same is true for the payoffs from deviating, the arguments remain valid in this case.

\subsubsection{Dynamics}

We know from (25) that for all $t \geq 2,\left(y_{p t+1}, y_{m t+1}\right)=\left(\gamma_{H}-\eta y_{p t}, \gamma_{L}-\eta y_{m t}\right)$. This is a linear system of difference equations which can be written in matrix form as

$$
\left[\begin{array}{l}
y_{p t+1} \\
y_{m t+1}
\end{array}\right]=\left[\begin{array}{cc}
-\eta & 0 \\
0 & -\eta
\end{array}\right]\left[\begin{array}{l}
y_{p t} \\
y_{m t}
\end{array}\right]+\left[\begin{array}{l}
\gamma_{H} \\
\gamma_{L}
\end{array}\right]
$$

By the same argument as in Proposition 1, this is a stable system and thus it converges to its steady state, which is (16).

\subsubsection{Surplus comparison}

The procedure for comparison follows the steps in the proof of Proposition 3. Surplus in the market equilibrium, denoted $S^{*}$, is as described in that proof. Surplus in the corrected equilibrium, denoted $S^{e}$, is the same except with the equilibrium values (45), (46), (48) and (49), the new equilibrium production levels $\mathbf{y}_{t}^{e}$, and the tax revenues

$$
\sum_{t=2}^{\infty} \delta^{t-1}\left(T_{y m} y_{m t}^{e}+T_{y p} y_{p t}^{e}\right)
$$

We may again write the difference in surplus as

$$
S^{*}-S^{e}=S_{T}^{*}-S_{T}^{e}+S_{s}^{*}-S_{s}^{e}
$$


where $S_{T}^{*}-S_{T}^{e}$ is the difference in surplus resulting from the taxes on young pets and $S_{s}^{*}-S_{s}^{e}$ is the difference resulting from the spaying subsidy. We can further show that

$$
\begin{gathered}
S_{T}^{*}-S_{T}^{e}=\frac{\left[\Delta_{p}(1-\delta)+\delta \gamma_{H}\right] \delta \eta(1-\alpha)}{(1-\delta)(1+\eta \alpha \delta)}\left\{\frac{T_{y p}}{(1+\delta \eta)}-\left(\xi_{o}+\rho\right)\right\}+ \\
\frac{\left[\Delta_{m}(1-\delta)+\delta \gamma_{L}\right] \delta \eta(1-\alpha)}{(1-\delta)(1+\eta \alpha \delta)}\left\{\frac{T_{y m}}{(1+\delta \eta)}-\left(\xi_{o}+\rho\right)\right\}
\end{gathered}
$$

where $\left(\Delta_{p}, \Delta_{m}\right)=\left(\gamma_{H}-\alpha H_{1}^{o}, \gamma_{L}-\alpha L_{1}^{o}\right)$. Using (18), (22), (23) and Assumption 6, we can show that $T_{y m} /(1+\delta \eta)-\left(\xi_{o}+\rho\right)$ is less than $s_{s} /(1+\delta \eta)$ and that $T_{y p} /(1+\delta \eta)-\left(\xi_{o}+\rho\right)$ is less than $\left(s_{s}-s_{s} / \delta x\right) /(1+\delta \eta)$. Thus, we can conclude that

$$
S_{T}^{*}-S_{T}^{e}<\frac{[\Delta(1-\delta)+\delta] \delta \eta(1-\alpha)}{(1-\delta)(1+\eta \alpha \delta)(1+\delta \eta)} s_{s}-\frac{\left[\Delta_{p}(1-\delta)+\delta \gamma_{H}\right] \delta \eta(1-\alpha)}{(1-\delta)(1+\eta \alpha \delta)(1+\delta \eta)} \frac{s_{s}}{\delta x}
$$

where $\Delta=1-\alpha\left(H_{1}^{o}+L_{1}^{o}\right)$. In addition, we have that

$$
S_{s}^{*}-S_{s}^{e}<-\frac{[\Delta(1-\delta)+\delta] \delta \eta(1-\alpha)}{(1-\delta)(1+\eta \alpha \delta)(1+\delta \eta)} s_{s}+\frac{\left[\gamma_{H}-(1-\delta) \alpha H_{1}^{o}\right] \delta \eta(1-\alpha)}{(1-\delta)(1+\eta \alpha \delta)(1+\eta \delta)} \frac{s_{s}}{\delta x}
$$

Combining (50) and (51) and using the definition of $\Delta_{p}$, we have that

$$
\begin{aligned}
S_{T}^{*}- & S_{T}^{e}+S_{s}^{*}-S_{s}^{e}<\frac{[\Delta(1-\delta)+\delta] \delta \eta(1-\alpha)}{(1-\delta)(1+\eta \alpha \delta)(1+\delta \eta)} s_{s}-\frac{[\Delta(1-\delta)+\delta] \delta \eta(1-\alpha)}{(1-\delta)(1+\eta \alpha \delta)(1+\delta \eta)} s_{s} \\
& +\frac{\left[\gamma_{H}-(1-\delta) \alpha H_{1}^{o}\right] \delta \eta(1-\alpha)}{(1-\delta)(1+\eta \alpha \delta)(1+\eta \delta)} \frac{s_{s}}{\delta x}-\frac{\left[\Delta_{p}(1-\delta)+\delta \gamma_{H}\right] \delta \eta(1-\alpha)}{(1-\delta)(1+\eta \alpha \delta)(1+\delta \eta)} \frac{s_{s}}{\delta x}=0
\end{aligned}
$$

as required. 
TABLE 1: RESULTS FROM CALIBRATION

\begin{tabular}{|c|c|c|c|}
\hline PARAMETER VALUES & & high $\theta$ & low $\theta$ \\
\hline ownership probability & $\alpha$ & 0.81 & 0.81 \\
\hline probability reaches old age & $\eta$ & 0.58 & 0.58 \\
\hline cost of spaying & $\mathrm{c}_{\mathrm{s}}$ & 100 & 100 \\
\hline resource cost of euthanasia & $\rho$ & 100 & 100 \\
\hline probability of mixed breed offspring & $\varphi$ & 0.21 & 0.21 \\
\hline discount factor & $\delta$ & 0.86 & 0.86 \\
\hline fraction of high types & $\gamma_{\mathrm{H}}$ & 0.58 & 0.58 \\
\hline fraction of low types & $\gamma_{\mathrm{L}}$ & 0.42 & 0.42 \\
\hline offspring in a litter & $x$ & 5.67 & 5.67 \\
\hline cost of litter & $c_{1}$ & 334 & 334 \\
\hline cost of breeding & r & 2151 & 2151 \\
\hline benefit from owning young mixed breed & $\beta$ & 2000 & 2000 \\
\hline benefit for high types from owning pure breed & $\theta$ & 2000 & 1000 \\
\hline deflation from owning new old pet & $\lambda$ & 0.75 & 0.75 \\
\hline psychic cost for young pet & $\xi_{\mathrm{y}}$ & 100 & 100 \\
\hline psychic cost for old pet & $\xi_{0}$ & 800 & 800 \\
\hline
\end{tabular}

OPTIMAL POLICIES

\begin{tabular}{|lccc|}
\hline tax on young mixed breeds & $\mathrm{T}_{\mathrm{ym}}$ & 632 & 632 \\
tax on young pure breeds & $\mathrm{T}_{\mathrm{yp}}$ & 632 & 488 \\
spaying subsidy & $\mathrm{S}_{\mathrm{s}}$ & 30 & 30 \\
\hline
\end{tabular}

WELFARE GAINS 
TABLE 2: ALTERNATIVE PARAMETER VALUES

HIGH BENEFITS FROM OWNERSHIP

\begin{tabular}{|c|c|c|c|}
\hline & & high $\theta$ & low $\theta$ \\
\hline benefit from owning young mixed breed & $\beta$ & 2500 & 2500 \\
\hline benefit for high types from owning pure breed & $\theta$ & 2500 & 1250 \\
\hline tax on young mixed-breeds & $T_{y m}$ & 807 & 807 \\
\hline tax on young pure-breeds & $\mathrm{T}_{\mathrm{yp}}$ & 807 & 751 \\
\hline spaying subsidy & $\mathrm{s}_{\mathrm{s}}$ & 30 & 30 \\
\hline welfare gains & & 195 & 176 \\
\hline
\end{tabular}

\section{LOW BENEFITS FROM OWNERSHIP}

benefit from owning young mixed breed

benefit for high types from owning pure breed

tax on young mixed-breeds

\begin{tabular}{ccc|} 
& high $\theta$ & low $\theta$ \\
$\beta$ & 1500 & 1500 \\
$\theta$ & 1500 & 750 \\
$\mathrm{~T}_{\mathrm{ym}}$ & 456 & 456 \\
$\mathrm{~T}_{\mathrm{yp}}$ & 456 & 224 \\
$\mathrm{~S}_{\mathrm{s}}$ & 30 & 30 \\
& 282 & 194 \\
\hline
\end{tabular}

tax on young pure-breeds

spaying subsidy 194

welfare gains

HIGH PSYCHIC COSTS

psychic cost for young pet

psychic cost for old pet

tax on young mixed-breeds

tax on young pure-breeds

spaying subsidy

welfare gains

\begin{tabular}{ccc|} 
& high $\theta$ & low $\theta$ \\
\hline$\xi_{\mathrm{y}}$ & 125 & 125 \\
$\xi_{\mathrm{o}}$ & 1000 & 1000 \\
$\mathrm{~T}_{\mathrm{ym}}$ & 639 & 639 \\
$\mathrm{~T}_{\mathrm{yp}}$ & 639 & 493 \\
$\mathrm{~S}_{\mathrm{s}}$ & 37 & 37 \\
& 318 & 264 \\
\hline
\end{tabular}

LOW PSYCHIC COSTS

psychic cost for young pet

psychic cost for old pet

tax on young mixed-breeds

tax on young pure-breeds

spaying subsidy

welfare gains

\begin{tabular}{ccc} 
& high $\theta$ & low $\theta$ \\
$\xi_{\mathrm{y}}$ & 75 & 75 \\
$\xi_{\mathrm{o}}$ & 600 & 600 \\
$\mathrm{~T}_{\mathrm{ym}}$ & 624 & 624 \\
$\mathrm{~T}_{\mathrm{yp}}$ & 624 & 482 \\
$\mathrm{~S}_{\mathrm{s}}$ & 22 & 22 \\
& 159 & 105 \\
\hline
\end{tabular}

